\title{
Smooth coalgebra: testing vector analysis
}

\author{
DUSKO PAVLOVIC PAnd BERFRIED FAUSER $^{\ddagger}$ \\ ${ }^{\dagger}$ Information and Computer Sciences Department, University of Hawaii, HI 96822 Honolulu, USA \\ Email: dusko@hawaii.edu \\ ${ }^{\ddagger}$ Mathematisch- Naturwissenschaftliche Sektion, University of Konstanz, 78464 Konstanz, Germany \\ Email: bertfried.fauser@gmail.com
}

Received 28 March 2013; revised 23 February 2015

\begin{abstract}
Processes are often viewed as coalgebras, with the structure maps specifying the state transitions. In the simplest case, the state spaces are discrete, and the structure map simply takes each state to the next states. But the coalgebraic view is also quite effective for studying processes over structured state spaces, e.g. measurable, or continuous. In the present paper, we consider coalgebras over manifolds. This means that the captured processes evolve over state spaces that are not just continuous, but also locally homeomorphic to normed vector spaces, and thus carry a differential structure. Both dynamical systems and differential forms arise as coalgebras over such state spaces, for two different endofunctors over manifolds. A duality induced by these two endofunctors provides a formal underpinning for the informal geometric intuitions linking differential forms and dynamical systems in the various practical applications, e.g. in physics. This joint functorial reconstruction of tangent bundles and cotangent bundles uncovers the universal properties and a high-level view of these fundamental structures, which are implemented rather intricately in their standard form. The succinct coalgebraic presentation provides unexpected insights even about the situations as familiar as Newton's laws.
\end{abstract}

\section{Introduction}

\subsection{Idea: 'Gedankenexperiments' with algebras and coalgebas}

The coalgebraic view of processes is based on capturing the state transitions by the coalgebra structure maps. Since the coalgebra homomorphisms then preserve and reflect the state transitions, they capture the observable behaviours, and the elements of the final coalgebras correspond to the equivalence classes of indistinguishable behaviours. The main utility of the coalgebraic theory of processes has been drawn from this correspondence (Rutten 2000).

However, what is observable, and which behaviours can be distinguished, is not always determined just by the transition systems, i.e. by the coalgebraic specifications alone, like it is in the special case of concurrent processes modulo strong bisimulation (Aczel 1988; Pavlovic 1995). Both in system design and in theoretical analyses, behaviours are usually specified in various testing frameworks, along the lines of E.F. Moore's seminal 'Gedankenexperiments' paper (Moore 1956). For example, in the theory of computation, a process can be specified as a Turing machine, or as a finite state automaton, and such specifications can be naturally presented as coalgebras. But to specify how this process processes data, i.e. how does a state machine compute, and what does it mean that 
it accepts a language, we must also say how it interacts with the words representing data. And the words are elements of algebras. The interactions between machines and words are Moore's testing correlations. A categorical version of testing correlations, where machines are presented as coalgebras, and tests as the elements of algebras, was studied in Pavlovic et al. (2006). Here, we lift the same framework to dynamical systems presented as coalgebras, and paths as the elements of algebras.

\subsection{Background: semantic connections of algebras and coalgebras}

It is essential to note that, in the testing approach to specifying behaviours, both coalgebras and algebras are parameters of the description, and neither side determines the other. For example, the whole linear time/branching time spectrum of essentially different computational behaviours (van Glabbeek 1990, 1993) can be described by fixing the family of coalgebras, and varying the test algebras. On the other hand, the Chomsky hierarchy of languages, as well as the hierarchy of complexity classes, can be described by fixing the family of tests, and varying the state machines, viz the corresponding coalgebras. Both approaches were aligned in Pavlovic et al. $(2006)^{\dagger}$, generalizing the earlier application in Pavlovic (2001). Such loose semantical connections of algebras and coalgebras, as two independent dimensions of semantical descriptions, distinguish the testing frameworks used in the present paper, and previously applied in Pavlovic (2001); Pavlovic et al. (2006, 2008, 2010); Pavlovic and Smith (2001, 2002), from the tight semantical connections of algebras and coalgebras, as arising on the two sides of a duality, and used in algebraic semantics of coalgebraic logic (Kupke et al. 2004, 2005; Kurz and Rosický 2012). The idea of testing is echoed more closely in the testing approach to the equivalence of concurrent processes (Bernardo et al. 2012; De Nicola and Hennessy 1984), and the two-dimensional approach is implemented in terms of algebras and coalgebras in the categorical approach to Structured Operational Semantics, which was developed as an extension of Denotational Semantics of monads (Klin 2007a, 2009; Turi and Plotkin 1997). However, while algebras and coalgebras do not completely constrain each other, and varying the algebras allows capturing the linear time/branching time spectrum, they were specified within the same category, with their semantical connection hardwired through distributivity requirements precluding, e.g. capturing the language hierarchies. On the other hand, semantic connections of algebras and coalgebras were studied in a wide variety of frameworks, and by a wide variety of techniques (Jacobs and Sokolova 2010; Kapulkin et al. 2012; Klin 2007b, to mention just a few), and it is possible that the language hierarchies, language acceptance, and computability and complexity concepts could have been captured in that framework if the community moved in that direction. The language acceptance relation and the trace equivalences have in fact been captured in Hasuo et al. (2007), but by combining coalgebras and monads, which seem to indirectly capture the underlying semantical connection. For example, capturing the linear

\footnotetext{
$\dagger$ The latter approach probably deserves a more detailed explanation, especially since its details had to be moved to the Appendix, and the Appendix got removed from the published version of the paper. The full submitted version remained available online.
} 
time-branching time spectrum in that framework seems quite a bit more involved than through testing. Last but not least, the general paradigm of testing, explicated by E.F. Moore, has been widely used before and independently of his work, not only in concurrency theory (Bernardo et al. 2012), but also, e.g. in functional analysis, as the foundation of theory of distributions (Schwartz 1950). In fact, the idea to apply testing in the context of manifolds, that we propose in the present paper, could have just as easily emerged from the theory of distributions as from the theory of coalgebras. A clearly coalgebraic view of differential geometry can be traced back to the 1970s work of Modungo and Stefani on the second tangent bundle and its mixed versions (Modungo and Stefani 1978). The naturality of the bundle structures was explicated in Kolar et al. (1993). A coalgebraic view of tangent bundles was proposed in Haghverdi et al. (2005), leading to the idea that dynamical systems could be viewed modulo the bisimulations relations. This however may suffer from too strong assumptions about a comonad structure on the tangent functor, see comments below.

A different categorical approach to tangent bundles, framed in the context of Synthetic Differential Geometry (SDG) (Kock 2006), goes back to Lawvere (1980) and Rosický (1984). The idea of SDG is to derive the important constructions of differential geometry from abstract categorical axioms. The analytic framework in which differential geometry is usually done is just one of the models of SDG. In order to open an alley towards differential geometry in non-standard categorical model categories, SDG attempts to extract and axiomatize what is essential for differential geometry, and to abstract away the inessential implementation details. This is, of course, a clean and exciting approach, but it involves researchers' design decisions what is essential in their theory, and what not. Many scientific discoveries, however, happen to emerge from the parts of scientific theories which have been previously thought to be inessential. This keeps scientific practice from being superseded by axiomatic theories. Nevertheless, axiomatic theories continue to play their role as well, and categorical axiomatics of differential geometry have in the meantime grown into a rich, active and useful research area (Blute et al. 2006, 2009; Cockett and Cruttwell 2014; Cockett and Seely 2011).

Last, we also mention the work on entwining structures, which has led our intuitions at various points, although its technical links with this work are less clear. Entwining structures are widely used in mathematical physics to study modules of quantum deformations (Caenepeel et al. 2002). A categorical description can be found in the papers by (Mesablishvili and Wisbauer 2010, 2011). Moerdijk's definition of a Hopf monad in Moerdijk (2002) seems to have been an important technical step in these analyses, with possible repercussions on our own work that remain to be explored.

\subsection{Outline of the paper}

In Section 2, we provide a general overview of testing correlations. In Section 3, we summarize the basic ideas about manifolds and their tangents. In Section 4, we apply testing correlations in the context of manifolds to provide the semantic reconstructions of the of the tangent bundle functors and of the cotangent bundle functors, spelled out in Theorem 4.11. The coalgebras for these endofunctors are the usual cross sections of 
the bundle projections, and thus respectively correspond to vector fields (or dynamical systems) and to differential forms. The testing correlations over manifolds thus provides a categorical view of the practice of integration of differential systems over differential forms. In Section 5, we spell out Newton's Second Law as an example of the coalgebraic treatment. Interestingly, the structural duality of the tangent and the cotangent bundles, displayed in the categorical treatment, immediately points beyond Newton, and into relativity theory. An overview of the standard definitions from vector analysis is provided in Appendix A.

\section{General testing framework}

We begin by reviewing the testing framework from Pavlovic et al. (2006).

\subsection{Idea}

Given a family of systems $\Sigma$, a family of tests $\Theta$ and a type $\Omega$ of observations, we call a map

$$
\Sigma \times \Theta \stackrel{\mathbb{T}}{\longrightarrow} \Omega
$$

a testing correlation, or just testing. The observation $\mathbb{T}(S, t)$ is often written in the infix form $S=t$. The observations can be boolean, like 'true'/'false', or 'pass'/ffail'; but they can also be quantities obtained from a measurement, e.g. in the interval $[0,1]$, or on the real line $\mathbb{R}$. Each test is assumed to yield a single observation. In the simplest case, we may use testing to distinguish a given system $S \in \Sigma$ from a reference system $R \in \Sigma$. The two systems are observably different if there is a test $b \in \Theta$ such that the observation $R \models b$ is different from the observation $S=b$. Otherwise, if the two systems induce the same observations for all tests, then they are observationally indistinguishable, and we write

$$
S \sim R \Longleftrightarrow \forall t \in \Theta .(S \models t)=(R \models t) .
$$

Developing this idea in Moore (1956), E.F. Moore suggested that minimal representations of automata can be built over the equivalence classes of their observationally indistinguishable states. This idea was elaborated categorically in Pavlovic et al. (2006), by identifying each equivalence class of systems that are observationally indistinguishable from $S \in \Sigma$ with the map $S \models(-): \Theta \rightarrow \Omega$. Such maps can be thought of as the observable behaviours of systems. The family $L$ of observable behaviours of systems from $\Sigma$ can thus be obtained as the image in $\Omega^{\Theta}$ of the mapping that sends each system $S$ to the induced function $S \models(-)$ from tests to observations:

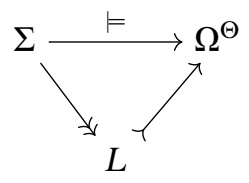

The main feature of this representation is that the elements of $L$, with a suitable coalgebraic structure, can be used to build the canonical minimal representatives of the behaviours of 
the systems in $\Sigma$, in so far as they are observable under testing by the tests from $\Theta$. Now, we outline the construction of this coalgebraic structure on $L$, as presented in Pavlovic et al. (2006).

\subsection{Semantic connections}

Let $\mathcal{S}$ be a category of 'spaces' and $\mathcal{T}$ be a category of 'types' or 'theories'. A semantic connection between $\mathcal{S}$ and $\mathcal{T}$ is simply a contravariant adjunction $M^{o p} \dashv P: \mathcal{S}^{o p} \rightarrow \mathcal{T}$. The idea is that a space $X \in \mathcal{S}$ is mapped to the type $P X$ of predicates over it, and that a theory $A \in \mathcal{T}$ is mapped to the space $M A$ of its models.

The examples abound. Stone duality motivates the logical view: $\mathcal{T}$ is the category of Boolean algebras, $\mathcal{S}$ is the category of Stone spaces, $M$ maps each Boolean algebra to its space of ultrafilters, which can be viewed as Boolean homomorphisms to 2, whereas $P$ sends each Stone space to its Boolean algebra of open sets, which can be viewed as continuous maps to 2 . If we restrict $\mathcal{S}$ from Stone spaces to the category of sets Set, viewed as topological spaces where every subset is open, then $\mathcal{T}$ restricts to the category of complete atomic Boolean algebras, which is equivalent to the opposite category of sets, which yields the self-adjunction of the powerset $\wp^{o p} \dashv \wp:$ Set $^{o p} \rightarrow$ Set as another example of a semantic connection. For a different example, let $\mathcal{S}$ be the lattice $\wp \mathbb{R}^{n}$ of sets of $n$-tuples of real numbers, $\mathcal{T}$ the lattice $\wp \mathbb{R}\left[x_{1}, \ldots x_{n}\right]$ of sets of real polynomials in $n$ variables and let $M$ map each set of polynomials to the $n$-tuples where they are all zero, whereas $P$ should map each set of $n$-tuples to all polynomials that vanish over it. When restricted to the fixed points of $M P$ and of $P M$, this semantic connection yields the duality of Zariski closed subsets of $\mathbb{R}^{n}$ and of radical ideals in $\mathbb{R}\left[x_{1}, \ldots x_{n}\right]$.

When they have enough limits and colimits, the two sides of a semantic connection share a dualizing object $\Omega$, which lives in both categories as the type $P 1$ and the space $M 1$ respectively. We can then construct the arrows

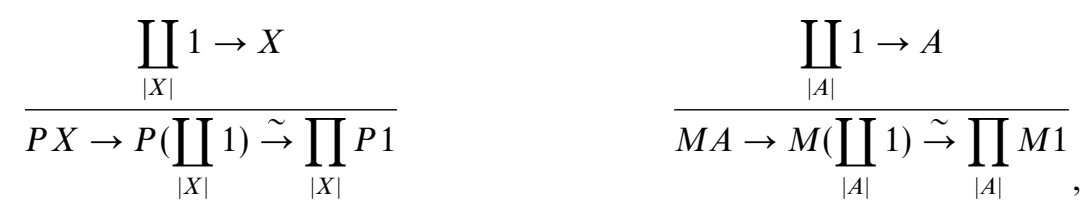

where $|C|=\mathrm{C}(1, C)$, and define the functors $\Omega^{X}:=\prod_{|X|} P 1$ and $\Omega^{A}:=\prod_{|A|} M 1$, to get the natural embeddings

$$
P X \succ \Omega^{X} \quad M A \nvdash \Omega^{A}
$$

\subsection{Testing coalgebras by process algebras}

Process logics are modal logics for describing the behaviour of computational processes (Harel et al. 2000; Milner 1989; Pratt 1976). With the modalities capturing the actions of a process, the formulas of process logics can be constructed as tests: a process satisfies a formula if and only if it passes the test that the formula represents. Viewed like this, semantics of process logics generalize the language acceptance definitions, used 
in the theory of computation to specify how automata and Turing machines compute. In the setting of semantic connections, semantics of process logics, including the various language acceptance definitions, can be captured as follows:

- Present processes as coalgebras in $\mathcal{S}$ for some endofunctor $G: \mathcal{S} \rightarrow \mathcal{S}$.

- Present process logics as algebras in $\mathcal{T}$ for some endofunctor $F: \mathcal{T} \rightarrow \mathcal{T}$.

- Specify the $F$-algebra semantics of $G$-coalgebras as a distributivity law $F P \stackrel{\lambda}{\rightarrow} P G$ : it lifts the final $G$-coalgebra $X \stackrel{\xi}{\rightarrow} G X$ into an $F$-algebra $F P X \stackrel{\lambda}{\rightarrow} P G X \stackrel{P \xi}{\rightarrow} P X$.

- The $F$-algebra testing of $G$-coalgebras is realized as the algebra homomorphism from the initial $F$-algebra $F A \stackrel{\alpha}{\rightarrow} A$ to $F P X \stackrel{\lambda}{\rightarrow} P G X \stackrel{P \xi}{\rightarrow} P X$ in $\mathcal{T}$.

- Transposed along the semantic connection, this algebra homomorphism induces in $\mathcal{S}$ a quotient $L$ of $X \stackrel{\xi}{\rightarrow} G X$, the finest (initial) among all of its quotients that embeds into $M A \stackrel{M \alpha}{\longrightarrow} M F A$.

- As an MFP-coalgebra, $L$ provides the canonical representatives of $F$-observable behaviours of $G$-processes.

This procedure is our formalization 'in the small' of the idea of Equation (2). The coalgebra $X \stackrel{\xi}{\rightarrow} G X$ represents the family of systems $\Sigma$, the algebra $F A \stackrel{\alpha}{\rightarrow} A$ represents the family of tests $\Theta$ and the elements of the dualizing object $\Omega$ are the available observations. This formalization was elaborated in Pavlovic et al. (2006). We quote the main theorem from that paper, omitting the considerations regarding the initiality of $F A \stackrel{\alpha}{\rightarrow} A$ and the finality of $X \stackrel{\xi}{\rightarrow} G X$.

Theorem 2.1. For the 'model/predicate' adjunction $M^{o p} \dashv P: \mathcal{S}^{o p} \rightarrow \mathcal{T}$ and endofunctors $G: \mathcal{S} \rightarrow \mathcal{S}$ and $F: \mathcal{T} \rightarrow \mathcal{T}$ and a distributive law $\lambda: F P \rightarrow P G$, the following holds:

a. The predicate functor $P: \mathcal{S}^{o p} \rightarrow \mathcal{T}$ lifts to $\hat{P}:\left(\mathcal{S}_{G}\right)^{o p} \rightarrow{ }_{F} \mathcal{T}$

$$
\frac{X \stackrel{\xi}{\longrightarrow} G X}{\hat{P} \xi: F P X \stackrel{\lambda}{\longrightarrow} P G X \stackrel{P \xi}{\longrightarrow} P X} \text { LIFT }
$$

b. $\hat{P}$ has in general no adjoint, but there is a correspondence

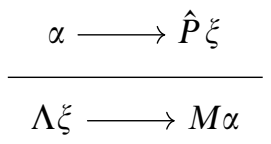


where $\Lambda: \mathcal{S}_{G} \rightarrow \mathcal{S}_{M F P}$ is the functor mapping the coalgebra $\xi: X \rightarrow G X$ to $X \stackrel{\xi}{\rightarrow} G X \stackrel{\lambda^{\prime}}{\rightarrow} M F P X . \lambda^{\prime}$ is the twisted distributivity law.
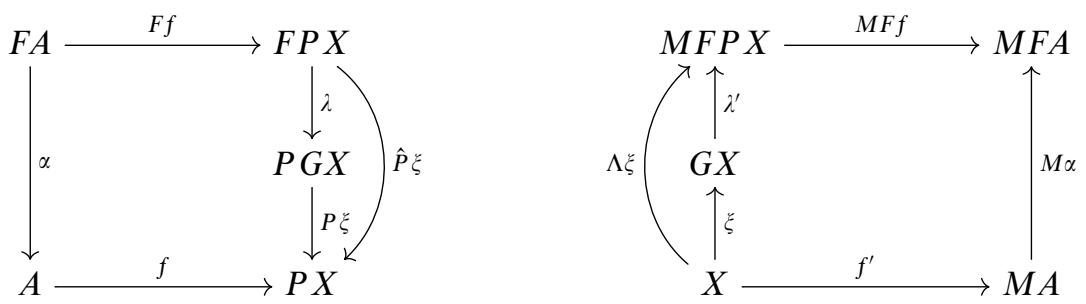

c. If $\mathcal{T}$ is a regular category, and $F: \mathcal{T} \rightarrow \mathcal{T}$ preserves reflexive coequalizers, then ${ }_{F} \mathcal{T}$ is a regular category. Every $F$-algebra homomorphism $\alpha \stackrel{f}{\rightarrow} \hat{P} \xi$ has a regular epi-mono factorization.

d. If $\mathcal{S}^{o p}$ is a regular category, and $M F P$ preserves weak pull backs, then every twisted coalgebra homomorphism $f^{\prime}: \Lambda \xi \rightarrow M \alpha$ has a regular epi-mono factorization, which induces a coalgebra $\ell: L \rightarrow M F P L$ as the image of $\Lambda \xi$

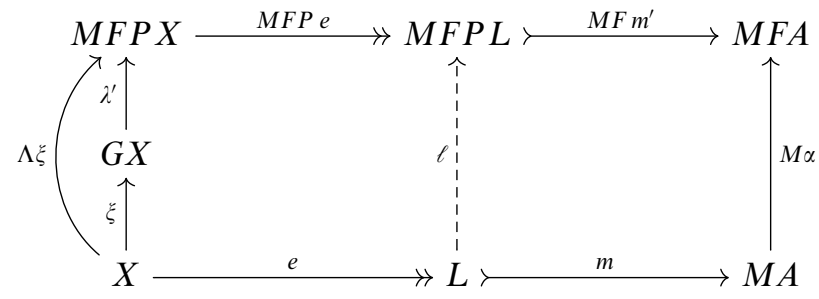

2.3.1. Examples. Testing a standard coalgebraic presentation of automata (e.g. Rutten (2000)) by linear process formulas yields regular languages as the behaviours in $L$. The MFP-coalgebra structure is induced by the language derivative. Testing the same family of automata by a process logic with branching yields the behaviours modulo the various bisimulation concepts from the branching time spectrum (van Glabbeek 1990, 1993). Testing a coalgebraic version of pushdown automata or Turing machines by linear process formulas yields context-free languages, respectively recursively enumerable languages as the behaviours in $L$, again with the language derivative as the coalgebra structure. The details are in the Appendix of Pavlovic et al. (2006) ${ }^{\dagger}$. The approach also extends to nondeterministic and probabilistic systems. The reason why testing coalgebras by algebras turns out to be both so versatile, and also simpler than most of the purely coalgebraic approaches to process semantics, is that it is, in a sense, just a categorical formalization of the familiar practice of defining computational models by specifying state machines on one hand and a language acceptance relation on the other. It should be noted, though, that, e.g. the purely coalgebraic approaches to trace semantics along the lines of Hasuo et al. (2007) are certainly more appropriate for some purposes than

\footnotetext{
$\dagger$ As mentioned earlier, the Appendix was omitted from the published volume. The original version of the paper is available online.
} 
our testing trace semantics, while the non-coalgebraic approaches to computability and complexity, e.g. through a monoidal computer (Pavlovic 2013), are more convenient for other purposes than the coalgebraic Turing machines of Pavlovic et al. (2006). And finally, while all examples discussed so far are concerned with computation, our goal in the present paper is to perhaps make a further step following the ideas of Pavlovic and Escardó (1998), and to provide a coalgebraic reconstruction of some concepts from mathematical analysis.

2.3.2. Vector calculus by testing. It turns out that both vector fields and differential forms can be reconstructed using a simple semantic connection where $\mathcal{S}=\mathcal{T}$ is the category of manifolds viewed within a topos, and $M X=P X=\mathbb{R}^{X}$, i.e. the dualizing object, is an object of reals. In this setting, vector fields (or dynamical systems) will be viewed as processes, presented as coalgebras, whereas differential forms can be viewed as tests, and they can be presented as algebras.

\section{Elements of vector calculus}

\subsection{Differentials}

One way to establish that two functions behave similarly at a point is to define their approximation relation.

Definition 3.1. Let $X$ and $Y$ be metric spaces with the distance functions $d_{X}: X \times X \rightarrow \mathbb{R}_{+}$ and $d_{Y}: Y \times Y \rightarrow \mathbb{R}_{+}$, and let $f, g: X \rightarrow Y$ be continuous functions. We say that $f$ and $g$ approximate each other at $c \in X$ whenever the distance between $f$ and $g$ vanishes at $c$, i.e.

$$
f \underset{c}{\sim} g \Longleftrightarrow \lim _{x \rightarrow c} \frac{d_{Y}(f(x), g(x))}{d_{X}(x, c)}=0 .
$$

For convenience, we list the axioms for metrics in the Appendix. Differential calculus really begins when $X$ and $Y$ are not just metric spaces, but also vector spaces, so that the question of linear approximation can be asked. To tie the metric and the linear structure together, it is usually required that the conditions

$$
d(x+u, y+u)=d(x, y) \quad d(r x, r y)=|r| \cdot d(x, y)
$$

hold for all vectors $u$ and all scalars $r$. Such a distance function on a metric space $E$ can then equivalently be specified using a norm $\|-\|: E \rightarrow \mathbb{R}_{+}$, which is related with $d: E \times E \rightarrow \mathbb{R}_{+}$by

$$
\|x\|=d(x, 0) \quad d(x, y)=\|x-y\| .
$$

The axioms for the norm are also in the Appendix. This brings us into the realm of normed vector spaces. They are a staple of functional analysis, at least when they are complete under the induced topology: complete normed vector spaces are known as Banach spaces. Since our goal here is to reconstruct the concepts of vector calculus from the minimal structural assumptions, we ignore the completeness requirement for the moment. Normed 
vector spaces, and their conceptual ancestors and origins were studied thoroughly and exhaustively ${ }^{\dagger}$ in Grothendieck's thesis (Grothendieck 1973). The norm and the linear structure suffice for defining the notion of differential.

Definition 3.2. Let $X$ and $Y$ be normed vector spaces. The norm structure determines the set $C(X, Y)$ of continuous maps, whereas the vector space structure determines the set $L(X, Y)$ of linear maps. The differential of $f \in C(X, Y)$ is the partial operation

$$
\text { D } f: X \rightarrow L(X, Y)
$$

such that the linear operator $\mathbf{D} f(c)$, whenever it exists, approximates the difference $f(x)-f(c)$, in the sense

$$
f(x)-f(c) \underset{c}{\sim} \mathbf{D} f(c)(x-c),
$$

where $x$ is a variable and $c$ a constant, i.e. by unfolding Equation (6)

$$
\lim _{x \rightarrow c} \frac{\|f(x)-f(c)-\mathbf{D} f(c) \cdot(x-c)\|}{\|x-c\|}=0 .
$$

The function $f$ is differentiable if $\mathbf{D} f$ is defined for all $c \in X$. The set of differentiable functions is written $C^{1}(X, Y)$.

The differentials can in fact be viewed as the linearizations of the equivalence classes modulo the approximation relation (6). The following lemma spells this out formally. The proof is a standard exercise with the norm.

Lemma 3.3. Let $f, g \in C^{1}(X, Y)$ be differentiable functions between normed vector spaces. Then, $f \underset{c}{\sim} g$ holds if and only if $\mathbf{D} f(c)=\mathbf{D} g(c)$ and $f(c)=g(c)$.

Whenever $L(X, Y)$ is metrizable, and thus a normed vector space, then the differential construction can be iterated, i.e. applied to the differential itself whenever it is continuous and thus $\mathbf{D} f \in C(X, L(X, Y))$, leading to

$$
\mathbf{D}^{2} f=\mathbf{D}(\mathbf{D} f): X \rightarrow L(X, L(X, Y)) \cong L(X \otimes X, Y) .
$$

Note that $L(X, Y)$ is not metrizable in general, but the peculiar situations when it is not have been characterized in Mackey's work, and clearly spelled out in (Grothendieck 1973, Ch. 3). In general, whenever $\mathbf{D}^{i} f \in C\left(X, L\left(X^{\otimes n}, Y\right)\right)$ for all $i=0,1, \ldots k$, we define

$$
\mathbf{D}^{k+1} f=\mathbf{D}\left(\mathbf{D}^{k} f\right): X \rightarrow L\left(X^{\otimes k+1}, Y\right)
$$

and write $f \in C^{k}(X, Y)$ when $f$ is $k$ times differentiable. The maps which are $k$ times differentiable for all $k$ are in

$$
C^{\infty}(X, Y)=\bigcap_{k=0}^{\infty} C^{k}(X, Y)
$$

$\dagger$ Grothendieck subsequently left the area, explaining to Malgrange: 'There is nothing more to do, the subject is dead.' (Jackson 2004, p. 1045) 
The convention is that $\mathbf{D}^{0} f=f$ and $C^{0}(X, Y)=C(X, Y)$, and of course $L\left(X^{\otimes 0}, Y\right)=$ $L(\mathbb{R}, Y)=Y$.

Definition 3.4. Denote by $\mathrm{NVS}_{k}$, the category where the objects are normed vector spaces and the morphisms are $k$ times differentiable maps, i.e. $\operatorname{NVS}_{k}(X, Y)=C^{k}(X, Y)$. The smooth morphisms are in $\operatorname{NVS}_{\infty}(X, Y)=C^{\infty}(X, Y)$.

\subsection{Manifolds}

Vector calculus is built upon the differentials as its main conceptual tool, and largely geared towards computing the linear approximations of differentiable processes. Since non-linear processes can only be linearly approximated locally, the spaces where processes are presented do not have a global linear structure, but only local linear structures. This means that every point has a neighbourhood homeomorphic with a normed vector space. This is the idea of manifold, going back to Riemann and Poincare, worked out in modern algebraic topology (Dold 1995).

Definition 3.5. A manifold is a topological space $M$ whose every point has a neighbourhood homeomorphic with convex open subset of a normed vector space. More precisely, a manifold consists of

- the underlying topological space $M$,

- a normed vector space $E$, called model space,

- an open cover $\mathcal{U}=\left\{U_{i} \mid i \in I\right\}$ of $M$, i.e. $\bigcup_{i \in I} U_{i}=M$,

— the open embeddings $U_{i} \stackrel{\varphi_{i}}{\longmapsto} E$ for all $i$, with convex images, called charts.

The set $\mathcal{A}=\left\{U_{i} \stackrel{\varphi_{i}}{\longmapsto} E \mid i \in I\right\}$ is called the atlas of the manifold $M$.

3.2.1. Remarks. Note that the definition did not mention the differential structure. It will be lifted from normed vector spaces to manifolds in a moment. The point of the current purely topological definition is that at each point $x \in M$, the chart $U \stackrel{\varphi}{\mapsto} E$ with $U \ni x$ induces a local linear structure; or more precisely, a local convex structure, since only the convex combinations of the points in the neighbourhood $U$ can be formed ${ }^{\dagger}$. This suffices for defining differentials. In most applications, each vector space $E$ is assumed to be given with a basis, and the charts thus induce the local coordinate systems within $M$. The spaces $E$ are usually even Euclidean, and thus presented as $\mathbb{R}^{n}$. For a moment, we stick with abstract normed vector spaces not so much for the sake of generality, as to emphasize what structure is really needed to get differential calculus going. Since normed vector spaces suffice for defining the differential, the charts induce the local differential structures. The only problem is that over the open sets, where the different charts may intersect, different differentials may be induced. The next definition preempts that.

$\dagger$ Other reasons and consequences of the convexity requirement on charts are spelled out in Grothendieck (1973, Ch. 2). 
Definition 3.6. A manifold $M$ is $k$ times differentiable if the charts induce the same $k$-tuple differentials over their intersections. More precisely, for any two charts $U_{0} \stackrel{\varphi_{0}}{\longmapsto} E$ and $U_{1} \stackrel{\varphi_{1}}{\longmapsto} E$ in the atlas of $M$, it is required that the homeomorphism

$$
\varphi_{0}\left(U_{0} \cap U_{1}\right) \stackrel{\varphi_{0}^{-1}}{\longrightarrow} U_{0} \cap U_{1} \stackrel{\varphi_{1}}{\longrightarrow} \varphi_{1}\left(U_{0} \cap U_{1}\right)
$$

is $k$ times differentiable, i.e. it is a $C^{k}$-diffeomorphism of the open convex images $\varphi_{0}\left(U_{0} \cap\right.$ $\left.U_{1}\right)$ and $\varphi_{1}\left(U_{0} \cap U_{1}\right)$ of $U_{0} \cap U_{1}$. A manifold is smooth if it is differentiable for all positive integers $k$, having $C^{\infty}$-diffeomorphisms.

A manifold is thus a topological space $M$ with a model space $E$ and an atlas $\mathcal{A}$ that lifts the differentials from $E$ to $M$. A morphism between $k$ times differentiable manifolds should preserve the $k$-tuple differentials. To align the continuity with the differentiability of the manifold morphisms, we need to slightly refine the notion of atlas.

Definition 3.7. The atlas $\mathcal{A}$ of a manifold $M$ is saturated if for every chart $U \stackrel{\varphi}{\rightarrow} E$ in $\mathcal{A}$ and every open subset $W$ of $U$, the restriction $W \hookrightarrow U \stackrel{\varphi}{\rightarrow} E$ is also an element of $\mathcal{A}$.

It is easy to see that every atlas has a unique saturation. A saturated atlas allows finding sufficiently small charts.

Definition 3.8. Let $M$ and $N$ be $k$ times differentiable manifolds with saturated atlases. A continuous map $f: M \rightarrow N$ is a $k$ times differentiable morphism if for every $x \in M$ and the neighbourhoods $V \in f(x)$ and $U \ni x$ such that $U \subseteq f^{-1}(V)$, with the charts $U \stackrel{\varphi}{\longmapsto} E$ and $V \stackrel{\psi}{\longmapsto} F$ the continuous map

$$
f_{\psi \varphi}: \varphi(U) \stackrel{\varphi^{-1}}{\rightarrow} U \stackrel{f}{\rightarrow} V \stackrel{\psi}{\rightarrow} \psi(V)
$$

is $k$ times differentiable, i.e. $f_{\psi \varphi}=\psi \circ f \circ \varphi^{-1} \in C^{k}(\varphi(U), \psi(V))$. This is illustrated in Figure 1.

The smooth morphisms are $k$ times differentiable for all $k$, i.e. they are the elements of $C^{\infty}(M, N)=\bigcap_{k=0}^{\infty} C^{k}(M, N)$.

The category of $k$ times differentiable manifolds and their morphisms is denoted $\operatorname{Man}_{k}$. The category of smooth manifolds and smooth morphisms is denoted $\operatorname{Man}_{\infty}$.

Modern vector calculus is largely built using differentiable manifolds (Abraham et al. 1988). The model spaces $E$ are usually assumed to be Banach spaces, i.e. normed vector spaces that are complete under the induced topology. However, the completeness plays no role in the definition of manifolds, or even of their tangents and vector fields. It is, of course, essential in solving differential equations and analysing dynamical systems, but conspicuously unnecessary for describing them. We shall thus deviate from the bulk of literature where manifolds are viewed as continuously varying Banach spaces, and work with manifolds as continuously varying normed vector spaces. Although perhaps less familiar, this view simplifies our categorical treatment. 


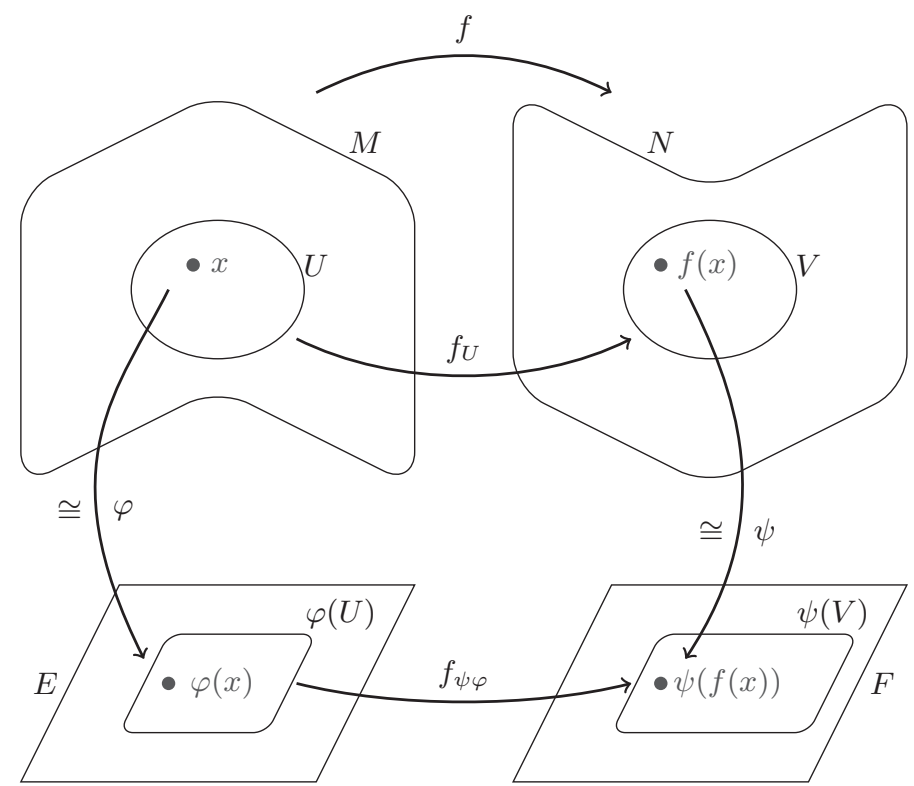

Fig. 1. $k$-times differentiable map $f: M \rightarrow N$.

\subsection{Tangent vectors}

The idea of a tangent vector is that it is the linear approximation of a curve at a given point. In vector calculus, the geometric idea of linear approximation is captured by the concept of the differential. If the curves in a normed vector space are viewed as the differentiable maps from $\mathbb{R}$, then we know from Lemma 3.3 that two curves $\varsigma, \chi \in C^{1}(\mathbb{R}, E)$ through the same point $e=\varsigma(0)=\chi(0)$ have the same differential if and only if $\varsigma \sim \chi$. The tangent vectors through a point $e$ of a normed vector space $E$ can thus be viewed as the equivalence classes modulo $\underset{0}{\sim}$. Lifting the differentials from normed vector spaces to manifolds, the tangent vectors on manifolds are defined in the same way, as illustrated in Figure 2.

Definition 3.9. A $k$ times differentiable curve or path on a manifold $M$ is a map $\varsigma \in$ $C^{k}(\mathbb{R}, M)$.

Definition 3.10. A tangent vector (or simply tangent) over a differentiable manifold $M$ is the equivalence class of paths in $M$ modulo the approximation relation. That is the set of all tangents over $M$ is collected in its tangent bundle

$$
T_{*} M=C^{1}(\mathbb{R}, M) / \underset{0}{\sim} .
$$

The equivalence classes of curves $[\varsigma]_{\sim} \in T_{*} M$ are often called linearizations of their elements.

The point of collecting the tangents over a manifold $M$ into a bundle is that the tangent bundle $T_{*} M$ is a manifold again, and that dynamical systems can be presented 


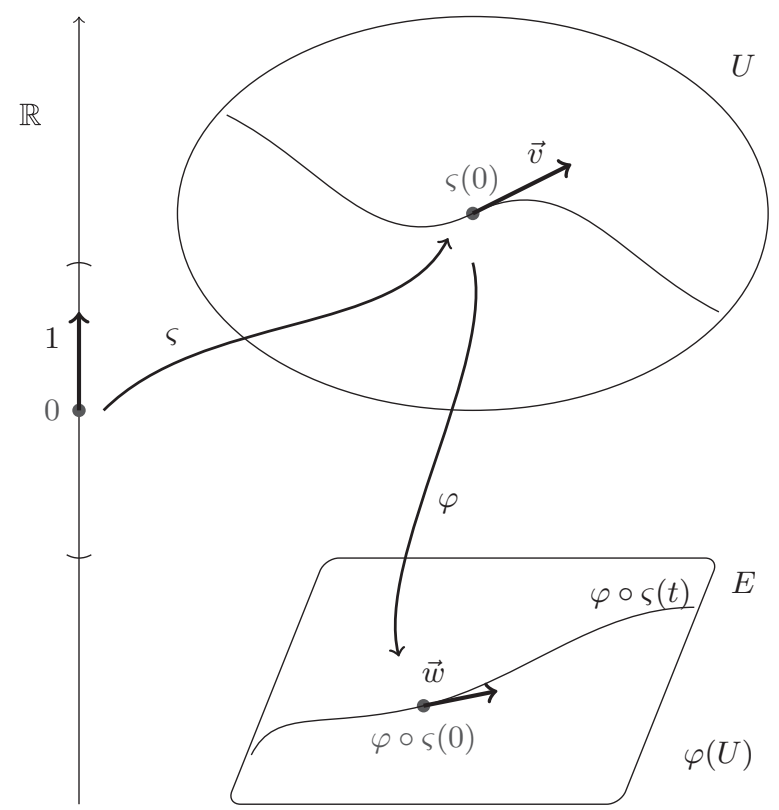

Fig. 2. A tangent vector on the manifold $M$ can be obtained in the form $\vec{v}=\mathbf{D} \varsigma(0) \cdot 1$, where $\varsigma: \mathbb{R} \rightarrow M$ is a suitable curve through the tangent point. By definition, the differential $\mathbf{D} \varsigma(0)$ in $M$ is the differential $\mathbf{D}(\varphi \circ \varsigma)(0)$ in $E$, where $U \stackrel{\varphi}{\longmapsto} E$ is a chart at $\varsigma(0)$.

as suitable manifold morphisms $M \rightarrow T_{*} M$. While this provides a useful high-level view of dynamical systems, the direct implementation of this idea requires working with the low-level representations of the tangents, and unfolding charts and atlases over and over again. To give the reader a flavour of this implementation, we sketch it in the rest of this section. In the next section, we propose a high-level view of tangent bundles, as behaviours recognized through testing.

3.3.1. The tangent bundle functor $T_{*}$. By definition, the elements of the tangent bundles are the equivalence classes of curves $[\varsigma] \sim$, where $\varsigma \in C^{1}(\mathbb{R}, M)$ is a representative. By mapping each tangent $[\varsigma] \sim$ to the point where it touches the manifold, we get the projection

$$
\begin{aligned}
\varepsilon: T_{*} M & \rightarrow M \\
{[\varsigma]_{\sim} } & \mapsto \varsigma(0)
\end{aligned}
$$

which is well defined by Lemma 3.3. We need to lift to $T_{*} M$ not just the topology, but also the atlas from $M$. Towards that goal, let us first consider a very special case.

Lemma 3.11. Consider an open set $U$ in a normed vector space $E$ as a manifold, with the obvious chart. Then, $T_{*} U \cong U \times E$.

Proof sketch Since the linearization of the path $\varsigma \in C^{1}(\mathbb{R}, E)$ at $\varsigma(0)$ is generally given by evaluating the differential $\mathbf{D}_{\varsigma}: \mathbb{R} \rightarrow L(\mathbb{R}, E)=E$ at 0 to get $\mathbf{D} \varsigma(0) \in E$, in this 
particular case we have

$$
\begin{aligned}
& T_{*} U \rightarrow U \times E \\
& {[\varsigma]_{\sim} \mapsto\langle\varsigma(0), \mathbf{D} \varsigma(0) \cdot 1\rangle,}
\end{aligned}
$$

where 1 is the unit vector at 0 in $\mathbb{R}$.

Proposition 3.12. $T_{*} M$ carries a canonical manifold structure which restricts to its open submanifolds

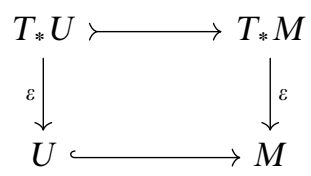

If $M$ is a $k$ times differentiable manifold, then $T_{*} M$ is $k-1$ times differentiable.

Proof sketch Suppose that the manifold $M$ is given with the atlas

$$
\mathcal{A}=\left\{U_{i} \stackrel{\varphi_{i}}{\rightarrow} E \mid i \in I\right\}
$$

We claim that

$$
T_{*} \mathcal{A}=\left\{U_{i} \times E \stackrel{\varphi_{i} \times E}{\longrightarrow} E \times E \mid i \in I\right\}
$$

is the atlas for $T_{*} M$ which restricts to $M$ 's submanifolds as claimed. For each linearization [ऽ] $\in T_{*} M$, the atlas $\mathcal{A}$ gives $\langle U, \varphi\rangle \in \mathcal{A}(\varsigma(0))$. In the neighbourhood of $q=\varsigma(0)$, the paths $\varsigma$ and $\varphi \circ \varsigma$ can be used interchangeably. But $\delta=\varphi \circ \varsigma: \mathbb{R} \rightarrow \varphi(U)$ is given by $\delta(t)=\varphi(\varsigma(0))+t \mathbf{D}(\varphi \circ \varsigma)(0) \cdot e$ and $\delta$ is completely determined by $\langle\varphi(\varsigma(0)), v\rangle$, with $v=\mathbf{D}(\varphi \circ \varsigma)(0) \cdot e=D \cdot e \in E$. Thus, the cylinder over $\phi(U)$ is a neighbourhood in $T_{*} M$, i.e. $\phi(U) \times E \in \mathcal{N}\left([\varsigma]_{\sim}\right)$.

Proposition 3.12 determines the object part of the functor $T_{*}: \operatorname{Man}_{k+1} \rightarrow \operatorname{Man}_{k}$. The arrow part is defined along the same lines.

Definition 3.13. Define the category of basic manifolds with $k$ times differentiable maps $\mathrm{BMan}_{k}$ as follows:

- the objects are pairs $\langle U, E\rangle$, where $E$ is a normed vector space, and $U \subseteq E$ is an open convex subset;

- the morphisms $\langle U, E\rangle \stackrel{f}{\rightarrow}\langle V, F\rangle$ are the $k$ times differentiable maps $f \in C^{k}(E, F)$ such that $f(U) \subseteq V$.

The category BMan $_{\infty}$ has the same objects, but the morphisms are smooth, i.e. $\operatorname{BMan}_{\infty}(M, N)=\bigcap_{k=0}^{\infty} \operatorname{BMan}_{k}(M, N)$. 
Proposition 3.14. The following mappings define a functor $T_{*}: \operatorname{BMan}_{k+1} \rightarrow \operatorname{BMan}_{k}$

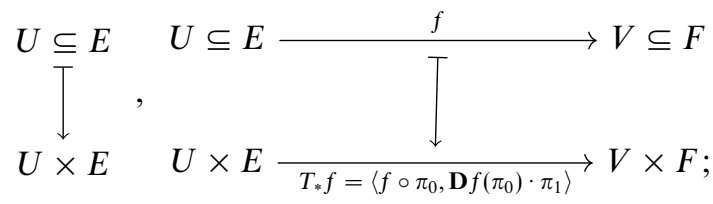

Restricted to the smooth morphisms in $\mathrm{Ban}_{\infty}$, the above construction gives the endofunctor $T_{*}: \operatorname{BMan}_{\infty} \rightarrow \operatorname{BMan}_{\infty}$.

Proof. We need to check that the arrow part of the construction is functorial. The fact that $T_{*}(\mathrm{id})=\mathrm{id}$ follows from Lemma 3.15 below. By unfolding the definition, which says that

$$
T_{*} f(q, v)=\langle f(q), \mathbf{D} f(q) \cdot v\rangle
$$

and using the chain rule (proved in the usual way) for the differential from Definition 3.2, we prove the claim

$$
\begin{aligned}
T_{*}(f \circ g)(q, v) & =\langle f(g(q)), \mathbf{D}(f \circ g)(q) \cdot v\rangle \\
& =\langle f(g(q)), \mathbf{D} f(g(q)) \cdot \mathbf{D} g(q) \cdot v\rangle \\
& =T_{*} f(g(q), \mathbf{D} g(q) \cdot v) \\
& =\left(T_{*} f \circ T_{*} g\right)(q, v) .
\end{aligned}
$$

Remark. This exhibits the fact that $T_{*}(f \circ g)=T_{*}(f) \circ T_{*}(g)$ is locally equivalent to the chain rule. The projection

$$
\begin{aligned}
\mathrm{BMan}_{k} & \rightarrow \mathrm{NVS}_{k} \\
\langle U, E\rangle & \mapsto E
\end{aligned}
$$

makes each $\mathrm{BMan}_{k}$ into a fibred category over $\mathrm{NVS}_{k}$. It is important to note that $T_{*}$ is not a lifting of the squaring functor

$$
\begin{aligned}
S: \operatorname{BMan}_{k} & \rightarrow \text { BMan }_{k} \\
X & \mapsto X \times X
\end{aligned}
$$

although the object part of $T_{*}$ defined above, mapping $U \subseteq E$ to $U \times E$, might suggest that it is. But the arrow part is obviously different: while the squaring functor maps $f \mapsto f \times f$, the second component of $T_{*} f$ is not $f$, but its linear approximation.

Lemma 3.15. $T_{*} f=f \times f$ if and only if $f$ is linear.

Proposition 3.16. The functors $T_{*}: \operatorname{BMan}_{k+1} \rightarrow \operatorname{BMan}_{k}$ have unique extensions to $T_{*}:$ $\operatorname{Man}_{k+1} \rightarrow \operatorname{Man}_{k}$ along the inclusions of basic manifolds into differentiable manifolds in 
general:

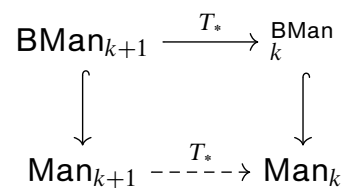

Proof. The object part of the extension maps the $k+1$ times differentiable manifolds $M$ to the tangent bundles $T_{*} M$ as described in Definition 3.10, with the manifold structure described in Proposition 3.12. Towards the arrow part, for any $f \in \operatorname{Man}_{k+1}(M, N)$ and any tangent $[\varsigma]_{\sim} \in T_{*} M$ represented by a differentiable curve $\varsigma: \mathbb{R} \rightarrow M$, define

$$
T_{*} f\left([\varsigma]_{\sim}\right)=[f \circ \varsigma]_{\sim} .
$$

The fact that this definition is sound, i.e. that $\varsigma \sim \varsigma^{\prime}$ over $M$ implies $f \circ \varsigma \sim f \circ \varsigma^{\prime}$ over $N$ follows from Lemma 3.3, using the chain rule. The fact that $T_{*} f$ is a $k$ times differentiable manifold homomorphism, i.e. in $\operatorname{Man}_{k}\left(T_{*} M, T_{*} N\right)$, follows from the fact that it restricts to $T_{*} f_{U} \in \operatorname{BMan}_{k}\left(T_{*} U, T_{*} V\right)$ for all corresponding charts $U \stackrel{\varphi}{\longmapsto} E$ of $M$ and $V \stackrel{\psi}{\mapsto} F$ of $N$ and the restriction $f_{U} \in \operatorname{BMan}_{k}(U, V)$ constructed as in Figure 1. They make the top trapezoid in the following diagram commutative.

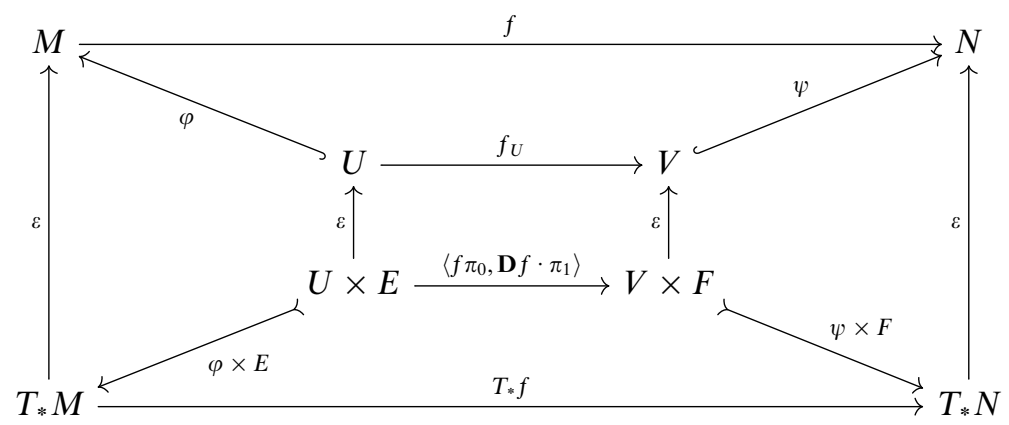

The trapezoids on the sides commute as in Equation (11), whereas the square in the middle commutes by Lemma 3.17, proved below. The bottom trapezoid then shows how $T_{*} f$, defined in Equation (12), restricts to a mapping on the charts as defined in Proposition 3.12. Tracking these restrictions around Figure 1 again, we see that $T_{*} f$ is locally glued from the differentials $\left\langle f \pi_{0}, \mathbf{D} f \cdot \pi_{1}\right\rangle$. If $f$ is $k+1$ times differentiable, then $\mathbf{D} f$ is $k$ times differentiable, and thus each restriction of $T_{*} f$ to a submanifold $T_{*} U=U \times E$ of $T_{*} M$ is $k$ times differentiable. Therefore, $T_{*} f$ must be $k$ times differentiable itself.

Lemma 3.17. The maps $\varepsilon_{M}: T_{*} M \rightarrow M$ defined in Equation (9), are natural when $M$ ranges over the basic manifolds $U$. Thus, $\varepsilon: T_{*} \rightarrow$ id is a natural transformation between the endofunctors on BMan $_{\infty}$. 
Proof. Unfolding the definitions

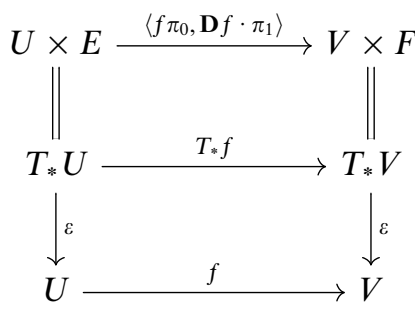

shows that projecting curves to their points $\varepsilon_{U}\left([\gamma]_{\sim}\right)=\varsigma(0)$, when restricted to basic manifolds $U$, boils down to the projections $\varepsilon=\pi_{0}: U \times E \rightarrow U$.

Just another look at diagram (13) gives

Corollary 3.18. The maps $\varepsilon_{M}: T_{*} M \rightarrow M$ form a natural transformation $\varepsilon: T_{*} \rightarrow$ id between endofunctors on $\operatorname{Man}_{\infty}$.

Proceeding along the same lines, we could further construct the natural transformation $\delta: T_{*} \rightarrow T_{*} T_{*}$, first for $T_{*}$ as an endofunctor on $\mathrm{BMan}_{\infty}$, where $T_{*} U \cong U \times E$, and thus

$$
\begin{aligned}
\delta: T_{*} U & \rightarrow T_{*} T_{*} U \\
\langle u, e\rangle & \mapsto\langle u, e, e, e\rangle
\end{aligned}
$$

and then extending this along Equation (11) to $T_{*}$ as an endofunctor on $\operatorname{Man}_{\infty}$. Remarkably, though, the natural transformation $T_{*} \stackrel{\delta}{\rightarrow} T_{*} T_{*}$ is coassociative, but not counitary with respect to $T_{*} \stackrel{\varepsilon}{\rightarrow}$ id and therefore does not make $T_{*}$ into a comonad. The reason is why it is not counitary, studied in more detail in Jubin (2012) is that all natural transformations between non-trivial powers of $T_{*}$ must preserve the zeros, as the trivial splitting of $T_{*} M \stackrel{\varepsilon}{\rightarrow} M$. Vector fields over a manifold $M$ can be viewed as the general splittings of $T_{*} M \stackrel{\varepsilon}{\rightarrow} M$, usually called cross sections; but they do not embed into $T_{*} M \stackrel{\delta}{\rightarrow} T_{*} T_{*} M$ as the cofree one, and they are thus not coalgebras of a monad, but just a special kind of coalgebras of the functor $T_{*}: \operatorname{Man}_{\infty} \rightarrow \operatorname{Man}_{\infty}$.

The whole process of functorializing tangent bundles, studied in the literature at many levels of generality and abstraction, can be repeated for cotangent bundles, leading to a cotangent bundle functor $T_{\#}: \operatorname{Man}_{\infty} \rightarrow \operatorname{Man}_{\infty}$ with a similar structure. While a tangent vector over a manifold $M$ is a $\sim$-equivalence class of curves $\varsigma: \mathbb{R} \rightarrow M$, a cotangent vector is a $\sim$-equivalence class of differentiable functions $f: M \rightarrow \mathbb{R}$. Just like vector fields can be presented as coalgebras $M \rightarrow T_{*} M$ such that the composite $M \rightarrow T_{*} M \stackrel{\varepsilon}{\rightarrow} M$ is the identity, differential forms can be presented as coalgebras $M \rightarrow T_{\#} M$ such that the composite $M \rightarrow T_{\#} M \stackrel{\varepsilon}{\rightarrow} M$ is the identity. Vector analysis is thus developed by using them together, and the cotangent bundle $T_{\#} M$ is usually defined as the dual of the tangent bundle $T_{*} M$. At a closer look, it turns out that both the tangent and the contangent vectors arise by testing, in the sense of Section 2 , of the differentiable functions $f: M \rightarrow \mathbb{R}$ along the curves $\varsigma: \mathbb{R} \rightarrow M$. This provides a high-level view of both constructions, which we present in the next section. 


\section{Tangent and cotangent testing}

\subsection{Manifolds as sheaves}

Let $M$ be a manifold with a saturated atlas $\mathcal{A}_{M}$. The fact that the atlas is saturated means that it can be presented as a presheaf, i.e. a functor mapping each open set $U \subseteq M$ to the set of charts over it, i.e.

$$
\begin{aligned}
\mathcal{A}_{M}: \mathcal{O}(M)^{o p} & \rightarrow \text { Set } \\
U & \mapsto\{U \stackrel{\varphi}{\rightarrow} E\},
\end{aligned}
$$

where $\mathcal{O}(M)$ is the set of opens in $M$, viewed as a partial order under inclusion ${ }^{\dagger}$. If the model space $E$ is given with some chosen coordinates $E \hookrightarrow \mathbb{R}^{X}$, then every $k$ times differentiable chart can be viewed as an $X$-tuple of $k$ times differentiable maps $U \rightarrow \mathbb{R}$, since there is the correspondence

$$
\frac{U \stackrel{\varphi}{\rightarrow} E \hookrightarrow \mathbb{R}^{X}}{U \times X \stackrel{\varphi \times X}{\longrightarrow} E \times X \rightarrow \mathbb{R}}
$$

By viewing each $k$ times differentiable chart as an $X$-tuple of $k$ times differentiable functions to $\mathbb{R}$, we can present the atlas $\mathcal{A}$ as the subfunctor of the sheaf of $k$ times differentiable functions

$$
\begin{aligned}
C^{k}(M): \mathcal{O}(M)^{o p} & \rightarrow \text { Set } \\
U & \mapsto C^{k}(U) .
\end{aligned}
$$

This is spelled out in Mac Lane and Moerdijk (1992, Section 2.3). When $M$ is a second countable Hausdorff space, then all of the structure of the manifold $M$ can be reconstructed from a subsheaf $\mathcal{A} \hookrightarrow C^{k}(M)$. Giving a $k$ times differentiable manifold on $M$ is equivalent to giving a subsheaf of $\mathcal{A} \hookrightarrow C^{k}(M)$ with the property that $U$ embeds into $\mathbb{R}^{\mathcal{A} U}$ for every $U$. Note, though, that the atlases of the manifolds presented like this are not only saturated, but also sheafified, i.e. they contain not only all subcharts of their charts, but also all unions of their charts that can be consistently glued together (Tennison 1975, 2.4). But since every saturated atlas has a sheafification (Mac Lane and Moerdijk 1992, Section 3.5), and the atlases that lead in this way to the same associated sheaf are just different presentations of diffeomorphic manifolds, this leads to no loss of generality. Sheaves thus provide a uniform presentation of manifolds, eliminating the irrelevant implementation details which allow different atlases for diffeomorphic, and thus indistinguishable manifolds. This is, of course, not an accident, since the concept of manifold was one of the guiding ideas behind the concept of sheaf (Leray 1945).

The main technical advantage of the sheaf presentation is that manifolds can now be studied in the rich environment of Grothendieck's toposes (Artin et al. 1972). This is where the tangent and cotangent bundle functors will emerge from testing.

$\dagger$ A partial order like $(\mathcal{O}(M), \subseteq)$ is a category with at most one morphism $U \rightarrow V$, which exists just when $U \subseteq V$ 
The idea of a topos of sheaves $\operatorname{Sh}(X)$ over a topological space $X$ is that it is a generalized view of the space $M$. From one angle, this view is supported by presenting sheaves as continuosly variable sets (Lawvere 1975), i.e. the contravariant functors $\mathcal{O}(X)^{o p} \rightarrow$ Set satisfying the sheaf requirement (Tennison $(1975,2.1)$ or Mac Lane and Moerdijk (1992, Section 2.1)), analogous to the above requirement that all consistent charts are glued together. The category $\operatorname{Sh}(X)$ is then viewed as a reflective subcategory of the functor category $\operatorname{Set}^{\mathcal{O}(X)^{o p}}$. From a different angle, sheaves over a space $X$ are equivalently presented as étale spaces, i.e. local homeomorphisms $E \rightarrow X$ (Tennison (1975, Section 2.3) or Mac Lane and Moerdijk (1992, Section 2.6)). The functor category $\operatorname{Sh}(X) \hookrightarrow \operatorname{Set}^{\mathcal{O}(X)^{o p}}$ is thus equivalent with the category of etale spaces $\mathcal{E} / X \hookrightarrow \mathrm{Esp} / X$, where Esp is the category of topological spaces. This latter presentation brings the sheaves over different spaces $X$ together in the category Top whose objects are étale spaces $E \stackrel{p}{\rightarrow} X$, while the morphisms are commutative squares formed by the parallel pairs of continuous maps $\langle e, b\rangle$ that make the square commute in the next diagram.

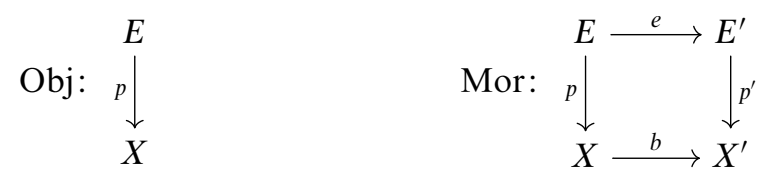

The category Top can be construed of as a crude version of Grothendieck's 'gros topos' Artin et al. (1972, IV.2.5), along the lines of Lawvere (1986).

Proposition 4.1. There is a faithful functor $\operatorname{Man}_{k} \longmapsto$ Top for every $k$. It is full for $k=0$.

Proof. Each manifold $M$ is presented as the étale space $E \rightarrow M$ corresponding to the sheaf $\mathcal{A}_{M}$ from Equation (15). The fact that the manifold morphisms are just the continuous maps that preserve the fibers follows directly from the definition of $\mathcal{A}_{M}$ as tracking the charts over $M$, and the construction of the points of $E \rightarrow M$ as the neighbourhood filters through these charts.

\subsection{Paths and tests}

The upshot of the presentation of manifolds as sheaves is that the category Man can thus be viewed as a subcategory of Top. Moreover, the forgetful functor Top $\rightarrow$ Esp, mapping each étale space $E \rightarrow X$ to its base space $X$, is a fibration, whose fibers are equivalent to the 'petit toposes' $\operatorname{Sh}(X)$.

Each of them contains the constant sheaf $\mathbb{R}$ of real numbers. The differentiable paths $\mathbb{R} \rightarrow M$ and the differentiable tests $M \rightarrow \mathbb{R}$ can now be internalized as sheaves.

Definition 4.2. Given a $k$ times differentiable manifold $M$, let the presheaves $\Sigma M, \Theta M$ : $\mathcal{O} M^{o p} \rightarrow$ Set be defined by

$$
\begin{aligned}
\Sigma M(U) & =\operatorname{Man}_{k}\left(\widehat{U} \times \mathcal{A}_{M}, \mathbb{R}\right) \\
\Theta M(U) & =\operatorname{Man}_{k}\left(\widehat{U} \times \mathbb{R}, \mathcal{A}_{M}\right) .
\end{aligned}
$$


Setting $\Sigma_{*} M=\Sigma M$ and $\Theta^{*} M=\Theta_{\#} M=\Theta M$ determines the object parts of the functors

$$
\Sigma_{*}, \Theta_{\#}: \operatorname{Man}_{k} \rightarrow \text { Top and } \Theta^{*}: \operatorname{Man}_{k}^{o p} \rightarrow \text { Top. }
$$

Given a $k$ times differentiable map $h \in \operatorname{Man}_{k}(M, N)$, the arrow parts will be defined as follows:

$$
\begin{aligned}
\Sigma_{*} h(U): \Sigma_{*} M(U) & \rightarrow \Sigma_{*} N(U) \\
\varsigma & \mapsto h \circ \varsigma \\
\Theta^{*} h(U): \Theta^{*} N(U) & \rightarrow \Theta^{*} M(U) \\
t & \mapsto t \circ\langle\mathrm{id}, h\rangle \\
\Theta_{\#} h(U): \Theta_{\#} M(U) & \rightarrow \Theta_{\#} N(U) \\
s & \mapsto \Theta_{\#} h(U)_{s}(u, y)=\bigvee_{\varepsilon>0} \bigwedge_{\|h(x)-y\| \leqslant \varepsilon} s(u, x),
\end{aligned}
$$

where $\varsigma \in \operatorname{Man}_{k}(\widehat{U} \times \mathbb{R}, M)$ is a path in $M$ whereas $t \in \operatorname{Man}_{k}(\widehat{U} \times N, \mathbb{R})$ and $s \in$ $\operatorname{Man}_{k}(\widehat{U} \times M, \mathbb{R})$ are tests over $x \in M$ and $y \in N$.

Remark. Since the inclusion $\operatorname{Sh}(X) \hookrightarrow \operatorname{Set}^{\mathcal{O}(X)^{o p}}$ has a left exact left adjoint (Mac Lane and Moerdijk 1992, Section 3.5), ${ }^{\dagger}$ the exponents of $\operatorname{Sh}(X)$ are created in $\operatorname{Set}^{\mathcal{O}(X)^{o p}}$. For $A, B \in \operatorname{Sh}(X)$, the exponent is thus

$$
B^{A}(U)=\operatorname{Sh}(\widehat{U} \times A, B) .
$$

Comparing this with Equations (18) and (19), it is clear that $\Theta M$ is a subsheaf of the sheaf $\mathbb{R}^{M}$, whereas $\Sigma M$ is a subsheaf of $M^{\mathbb{R}}$, where we abuse notation and write $M$ for the sheaf $\mathcal{A}_{M}$, representing the manifold $M$.

4.2.1. Convention. To simplify notation, when reasoning about sheaves and their morphisms, we often leave the variation over the open sets $U \subseteq M$ implicit. This is formally justified not only for the global constructions, but also for the local constructions that are expressible in the internal language of a topos Mac Lane and Moerdijk (1992, Ch. VI).

Proposition 4.3. The inclusions

$$
\Sigma_{*} M \hookrightarrow M^{\mathbb{R}} \quad \text { and } \quad \Theta^{*} M \hookrightarrow \mathbb{R}^{M}
$$

are natural in $M$, where the functors

$$
(-)^{\mathbb{R}}: \operatorname{Man}_{k} \rightarrow \text { Top } \quad \text { and } \quad \mathbb{R}^{(-)}: \operatorname{Man}_{k}^{o p} \rightarrow \text { Top }
$$

are obtained by restricting the exponentiation from the fibres of Top.

The proof of the preceding proposition boils down to unfolding the arrow part of the restriction $\mathbb{R}^{(-)}: \operatorname{Man}_{k}^{o p} \rightarrow$ Top of the exponentiation functors in the toposes $\operatorname{Sh}(M)$,

\footnotetext{
$\dagger$ The topos theory results that we use are contained in most topos theory textbooks. The choice of the one that we keep referring to is entirely a matter of personal habit, and the reader may wish to consult any of the other presentations.
} 
which is identical to Equations (20) and (21). The next proposition, however, requires that we define the covariant version $\mathbb{R}_{\#}^{(-)}: \operatorname{Man}_{k} \rightarrow$ Top, which is just the exponentiation on the objects, but maps every differentiable morphism $h \in \operatorname{Man}_{k}(M, N)$ to the internal left adjoint of $\mathbb{R}^{h}$, i.e.

$$
\mathbb{R}_{\#}^{h} \dashv \mathbb{R}^{h}: \mathbb{R}^{N} \rightarrow \mathbb{R}^{M}
$$

Definition 4.4. The covariant functor $\mathbb{R}_{\#}^{(-)}: \operatorname{Man}_{k} \rightarrow$ Top maps each manifold $M$ to the sheaf $\mathbb{R}^{M}$, and maps each $h \in \operatorname{Man}_{k}(M, N)$ to

$$
\begin{aligned}
\mathbb{R}_{\#}^{h}(U): \mathbb{R}^{M}(U) & \rightarrow \mathbb{R}^{N}(U) \\
s & \mapsto \mathbb{R}_{\#}^{h}(U)_{s}(u, y)=\bigwedge_{\varepsilon \searrow 0} \bigvee_{\|h(x)-y\| \leqslant \varepsilon} s(u, x) .
\end{aligned}
$$

Lemma 4.5. The direct images along the morphisms with non-zero differentials are injective, modulo the approximation relation. More precisely, for every $h \in C^{1}(M, N)$ and every $c \in M$ such that $\mathbf{D} h(c) \neq 0$ holds

$$
\begin{gathered}
\mathbb{R}\left(\mathbb{R}_{\#}(h)_{t}\right) \underset{c}{\sim} t \\
T^{*}\left(T_{\#}(h)_{t}\right) \underset{c}{\sim} t,
\end{gathered}
$$

where we write $\mathbb{R}_{\#}(h)$ for $\mathbb{R}_{\#}^{h}$ and $\mathbb{R}(g)$ for $\mathbb{R}^{g}$.

Proof of 4.5. Equation (24) Omitting the sheaf variation along $U \subseteq M$, as it plays no role here, for all $z$ in the neighbourhood of $c$ holds

$$
\begin{aligned}
\mathbb{R}\left(\mathbb{R}_{\#}(h)_{t}\right)(z) & =\bigwedge_{\varepsilon \searrow 0} \bigvee_{\|h(x)-h(z)\| \leqslant \varepsilon} t(x) \quad \tilde{z} \\
& \sim \bigwedge_{z \searrow 0} \bigvee_{\mathbf{D} h(z)\|x-z\| \leqslant \varepsilon} t(x) \\
& =t(z) .
\end{aligned}
$$

Proposition 4.6. The inclusions

$$
\Theta_{\#} M \hookrightarrow \mathbb{R}_{\#}^{M}
$$

are natural in $M$ with respect to all continuously differentiable morphisms $h: M \rightarrow N$.

Proof. The claim is that the square

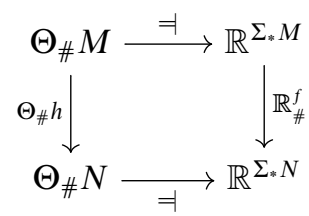


commutes for every continuously differentiable $h$. Going down and right gives

$$
\left(\Theta_{\#} h(t) \underset{N}{=} \tau\right)=\mathbf{D}\left(\bigwedge_{\varepsilon \searrow 0} \bigvee_{\|h(x)-\tau(r)\| \leqslant \varepsilon} t(x)\right)_{r}(0)
$$

where $t \in \Theta_{\#} M(U), \tau \in \mathbb{R}^{\Sigma_{*} N}(U)$ and the function under the differential operator varies over $r \in \mathbb{R}$. We omit the variable $u \in U$ on both sides, since the equation holds pointwise in it. Going right and down in Equation (26) gives

$$
\mathbb{R}_{\#}^{h}(U)_{\hat{t}}(\tau)=\bigwedge_{\varepsilon \searrow 0} \bigvee_{\|h(\varsigma(0))-\tau(0)\| \leqslant \varepsilon} \widehat{t}(\varsigma),
$$

where the supremum is taken over $\varsigma \in \Sigma_{*} M$ and $\widehat{t} \in \mathbb{R}^{\Sigma_{*} M}$ is defined to be

$$
\widehat{t}(\varsigma)=(t \underset{M}{=} \varsigma)=\mathbf{D}(t \circ \varsigma)(0) .
$$

Substituting $\varsigma$ into $\widehat{t}$ and putting Equations (27) and (28) together, the commutativity of Equation (26) thus boils down to

$$
\mathbf{D}\left(\bigwedge_{\varepsilon \searrow 0} \bigvee_{\|h(x)-\tau(0)\| \leqslant \varepsilon} t(x)\right)(0)=\bigwedge_{\varepsilon \searrow 0} \bigvee_{\|h(\varsigma(0))-\tau(0)\| \leqslant \varepsilon} \mathbf{D}(t \circ \varsigma)(0),
$$

the first supremum is taken over $x \in M$ and the second one over $\varsigma \in \Sigma_{*} M$. This equation holds as soon as $h, t$ and $\varsigma$ are continuously differentiable.

Remark. Note that $\bigwedge_{\varepsilon \succ 0} \bigvee_{\|h(x)-y\| \leqslant \varepsilon} g(x)$ is here just limsup $\operatorname{suph}_{y \rightarrow h(x)} g(x)$ and that the differential of a real function $y: \mathbb{R} \rightarrow \mathbb{R}$ depending on $x$ is just the slope of its tangent, i.e. the usual derivative $\mathbf{D} y=\frac{d y}{d x}$.

\subsection{Tangent testing correlation}

We are now in the position to define a testing correlation like in Equation (1) between the paths and the tests in a manifold $M$.

Definition 4.7. The testing correlation for differentiable manifold $M$ is the morphism $\mathbb{T}$ in $\operatorname{Sh}(M)$

$$
\begin{aligned}
\Sigma M \times \Theta M & \stackrel{\mathbb{T}}{\rightarrow} \mathbb{R} \\
\langle\mathbb{R} \stackrel{\varsigma}{\rightarrow} M, M \stackrel{t}{\rightarrow} \mathbb{R}\rangle & \mapsto \mathbf{D}(t \circ \varsigma)(0) .
\end{aligned}
$$

Lemma 4.8. The testing correlations are dinatural, in the sense that every differentiable manifold morphism $f: M \rightarrow N$ makes the following diagram commute.

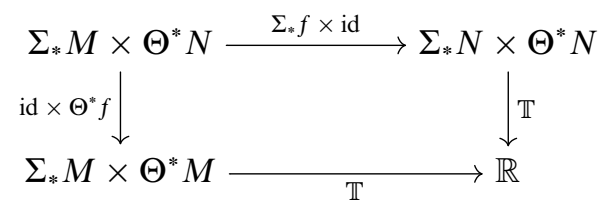


Proof. $\mathbb{T}\left(\varsigma, \Theta^{*} f(t)\right)=\mathbf{D}(t \circ f \circ \varsigma)(0)=\mathbb{T}\left(\Sigma_{*} f(\varsigma), t\right)$.

Recall from Section 2.1 that $\mathbb{T}(\varsigma, t)$ is also written in the infix forms

$$
\begin{aligned}
& \Sigma M \stackrel{\models}{\longrightarrow} \mathbb{R}^{\Theta M}: \quad \varsigma \mapsto(t \mapsto(\varsigma \models t)) \\
& \Theta M \stackrel{\Rightarrow}{\longrightarrow} \mathbb{R}^{\Sigma M}: \quad t \mapsto(\varsigma \mapsto(t=\varsigma))
\end{aligned}
$$

Lemma 4.8 now immediately implies:

Corollary 4.9. The testing correlations induce the natural transformations

$$
\Sigma_{*} \stackrel{\models}{\longrightarrow} \mathbb{R}^{\Theta^{*}} \quad \text { and } \quad \Theta^{*} \stackrel{\Rightarrow}{\longrightarrow} \mathbb{R}^{\Sigma_{*}} .
$$

Proceeding as in Equation (2), we define the 'observable behaviours' of the paths in $\Sigma M$ and of the tests in $\Theta M$ by the epi-mono factorizations in Top
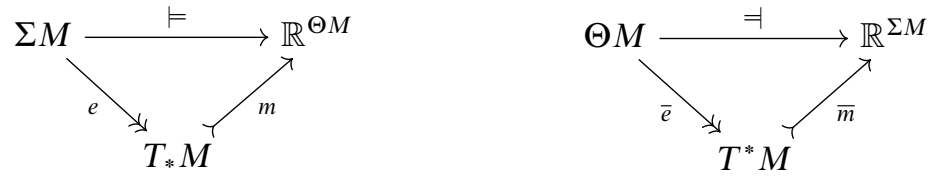

Proposition 4.10. If $M$ is a $k+1$ times differentiable manifold, then the sheaves $T_{*} M$ and $T^{*} M$ defined in Equation (30) are $k$ times differentiable manifolds: $T_{*} M$ is just the tangent bundle of $M$, whereas $T^{*} M$ is the cotangent bundle.

Proof. The claim that the quotient $T_{*} M$ defined in Equation (30) on the left is the same as the tangent bundle $T_{*} M$ defined in Section 3 formally means that for any two differentiable curves $\varsigma, \varsigma^{\prime} \in C^{k+1}(\mathbb{R}, M)$ through the same point $\varsigma(0)=\varsigma^{\prime}(0)$ holds

$$
\varsigma \underset{0}{\sim} \varsigma^{\prime} \Longleftrightarrow \forall t \in C^{k+1}(M, \mathbb{R}) . \mathbf{D}(t \circ \varsigma)(0)=\mathbf{D}\left(t \circ \varsigma^{\prime}\right)(0) \text {. }
$$

But this follows from Lemma 3.3.

The claim that the quotient $T^{*} M$ defined in Equation (30) on the right is the usual cotangent bundle manifold $T^{*} M$, i.e. that it consists of the cotangent vectors, dual to the tangent vectors in $T_{*} M$, is clear from the construction of the quotient $T^{*} M$, which is dual to the construction of $T_{*} M$.

\subsection{Main theorem}

Theorem 4.11. The factorizations in Equation (30) determine the functors

$$
T_{*}, T_{\#}: \operatorname{Man}_{\infty} \rightarrow \operatorname{Man}_{\infty} \quad \text { and } \quad T^{*}: \operatorname{Man}_{\infty}^{o p} \rightarrow \operatorname{Man}_{\infty}
$$

with $T_{\#} M=T^{*} M$ on the objects, and such that

- $T_{*}$ is a monad,

- $T_{\#}$ is a comonad and

- $T^{*}$ is self-adjoint. 
The proof of the theorem occupies the rest of this section.

4.4.1. The arrow part of $T_{*}$ and $T^{*}$. To define the arrow part of the functors $T_{*}$ and $T^{*}$, let $M$ and $N$ be smooth manifolds, and $f \in \operatorname{Man}_{\infty}(M, N)$ a smooth map between them. Consider the following diagrams:
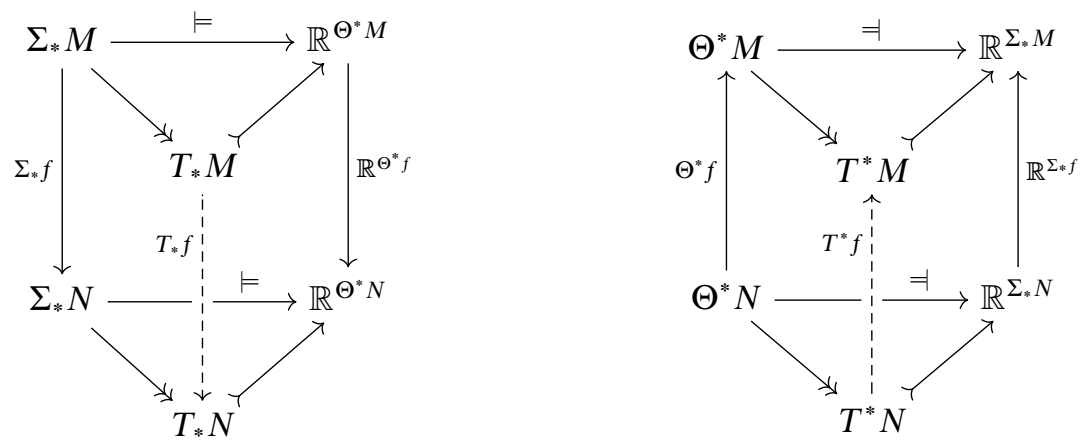

The back squares in both diagrams commute by Corrolary 4.9. The orthogonality of the monics and the epics in the factorizatons in Equation (30) then induces the unique maps $T_{*} f$ and $T^{*} f$. The functoriality of $\Sigma_{*}$ and $\Theta^{*}$ guarantees the functoriality of $T_{*}$ and $T^{*}$.

4.4.2. The arrow part of $T_{\#}$. To define the arrow part of the covariant cotangent bundle functor $T_{\#}$, consider

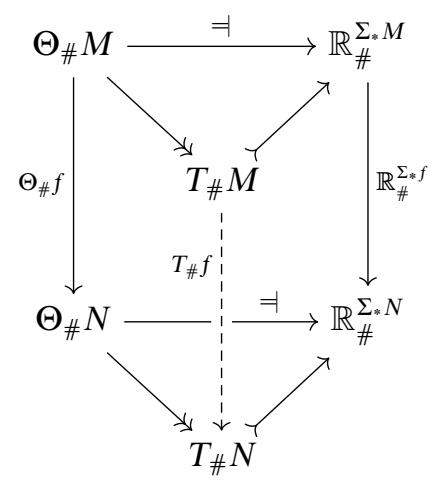

This time, the back square commutes by Proposition 4.6, and the functor thus depends on the direct images in the definitons of $\Theta_{\#}$ and $\mathbb{R}_{\#}^{\Sigma_{*}(-)}$. The uniqueness and the functoriality of the definition of $T_{\#} f$ follow from the same reasons as above. 
4.4.3. The bundle map $T_{*} \stackrel{\varepsilon}{\rightarrow} i d$. The bundle projection $\varepsilon: T_{*} M \rightarrow M$ is constructed using the orthogonality of the monics and the epis again

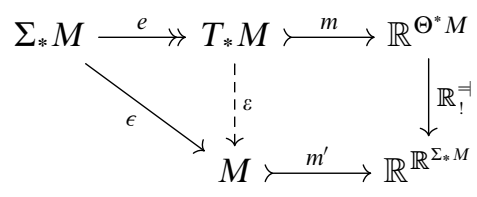

where the top is the definition of $T_{*} M$, the diagonal map is the projection

$$
\epsilon(\varsigma)=\varsigma(0)
$$

and the incusion $m^{\prime}$ is defined as follows:

$$
\frac{\mathbb{R}^{\Sigma_{*} M} \times M \stackrel{\mathbb{R}^{l} \times M}{\longrightarrow} \mathbb{R}^{M} \times M \longrightarrow \mathbb{R}}{M \stackrel{m^{\prime}}{\longrightarrow} \mathbb{R}^{\mathbb{R}^{\Sigma_{*} M}}}
$$

The map $\mathbb{R}_{\#}^{=}$is the image of the testing correlation along the covariant exponentiation functor from Equation (23), i.e.

$$
\frac{\Theta^{*} M \stackrel{=}{\leftrightarrows} \mathbb{R}^{\Sigma_{*} M}}{\mathbb{R}^{\Theta^{*} M} \underset{\mathbb{R}_{!}^{\ddagger}}{\stackrel{\mathbb{R}^{\ddagger}}{\leftrightarrows}} \mathbb{R}^{\mathbb{R}^{\Sigma * M}}}
$$

The natural transformation $\delta: T_{*} M \rightarrow T_{*} T_{*} M$ can be derived using the orthogonality of the factorization:

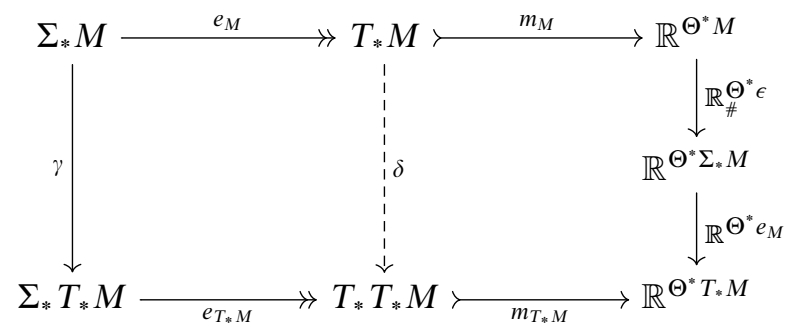

where

$$
\gamma(\varsigma)(y)=\varsigma+\mathbf{D} \varsigma(0) \cdot y
$$

and $\epsilon$ is as in Equation (33). This is not a comonad coevaluation, as it does not split $\varepsilon T_{*}$.

4.4.4. The monad structure $T_{*} T_{*} \stackrel{\mu}{\rightarrow} T_{*} \stackrel{\eta}{\leftarrow} i d$. The monad structure on $T_{*}$ is induced by — the bundle projections $\varepsilon: T_{*} M \rightarrow M$ from Section 4.4 .3 and the 
— the 'candidate units' $\eta_{\Sigma}: M \stackrel{\Sigma}{\rightarrow} M$, where $\eta_{\Sigma} x(r)=x$

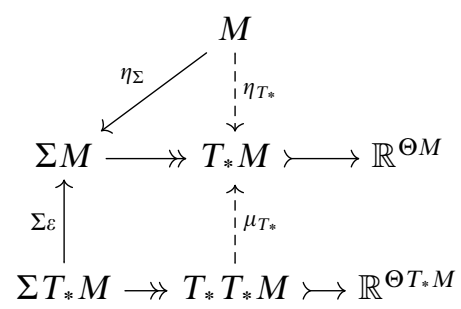

Remark. The monad structure over $T_{*}$ has been spelled out in the classical framework in Jubin (2012), and is studied in of SDG in Cockett and Cruttwell (2014).

4.4.5. The comonad structure $T_{\#} T_{\#} \stackrel{\delta}{\leftarrow} T_{\#} \stackrel{\varepsilon}{\rightarrow} I d$. Towards the definition of the bundle structure map $\varepsilon: T_{\#} M \rightarrow M$, define for each $x \in M$ the subsheaf $\Sigma_{x} M$ of $\Sigma_{*} M$ by setting

$$
\Sigma_{x} M=\left\{\varsigma \in \Sigma_{*} M \mid \varsigma(0)=x\right\} .
$$

Since $\Sigma_{*} M$ thus decomposes into the coproduct

$$
\Sigma_{*} M=\coprod_{x \in M} \Sigma_{x} M
$$

the cotangent bundle embeds into the product

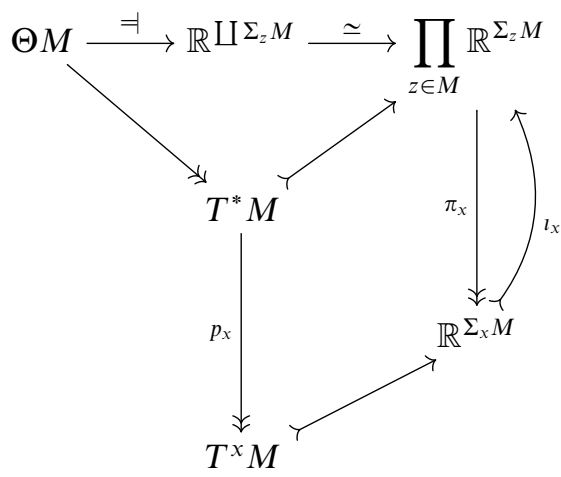

The quotient bundle $T^{x} M$ of $T^{*} M=T_{\#} M$ thus consists of the equivalence classes of the tests on $M$ that are indistinguishable by the paths through $x$. Note, however, that, since $\mathbb{R}$ is an injective object of $\operatorname{Sh}(M)$, the projections $\prod_{z \in M} \mathbb{R}^{\Sigma_{z} M} \stackrel{\pi_{x}}{\longrightarrow} \mathbb{R}^{\Sigma_{x} M}$ split by the injections $l_{x}: \mathbb{R}^{\Sigma_{x} M} \stackrel{l_{x}}{\rightarrow} \prod_{z \in M} \mathbb{R}^{\Sigma_{z} M}$. The splitting means that $\pi_{x} \circ l_{x}=\mathrm{id}$ holds. Intuitively, $l_{x}$ maps $\tau \in \mathbb{R}^{\Sigma_{x} M}$ to the family $l_{x}(\tau)=\left\langle\imath \tau_{z}\right\rangle_{z \in M}$, where $\imath \tau_{z}=\tau$ if $z=x$ and $\imath \tau_{z}=0$ otherwise. 
$T^{x} M$ is thus a submanifold of $\prod_{z \in M} \mathbb{R}^{\Sigma_{z} M}$, and hence

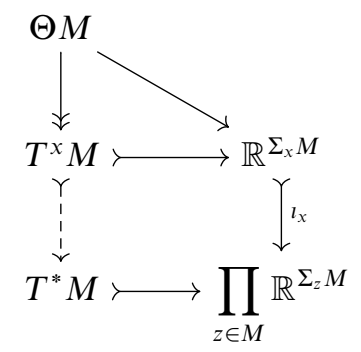

Since the inclusions $T^{x} M \hookrightarrow T^{*} M$ are jointly surjective, we have

$$
T^{*} M=\coprod_{x \in M} T^{x} M .
$$

Recalling that $T_{\#}$ is the covariant version of $T^{*}$, i.e. that $T_{\#} M=T^{*} M$ on the objects, we construct $\varepsilon: T_{\#} M \rightarrow M$ by projecting each $T^{x} M$ to $x \in M$. The coevaluation $\delta: T_{\#} M \rightarrow T_{\#} T_{\#} M$ follows by using the contravariant functors $\Theta^{*}$ and $\mathbb{R}^{\Sigma_{*}}$.

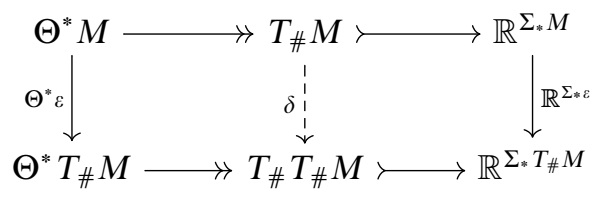

The naturality and the comonad conditions for the families

$$
M \stackrel{\varepsilon}{\leftarrow} T_{\#} M \stackrel{\delta}{\rightarrow} T_{\#} T_{\#} M
$$

follow from Lemma 4.5.

Remark. Just like the tangent bundle functor allowed a 'coevaluation candidate' $T_{*} \stackrel{\delta}{\rightarrow}$ $T_{*} T_{*}$ which turned out to be degenerate, the cotangent bundle allows a degenerate 'evaluation candidate' $T_{\#} T_{\#} \stackrel{\mu}{\rightarrow} T_{\#}$. While both candidacies fail, they may be pointing towards a structure that still waits to be understood.

4.4.6. $T^{*}$ is self-adjoint. The claim is that there is a correspondence

$$
\operatorname{Man}_{\infty}\left(M, T^{*} N\right) \cong \operatorname{Man}_{\infty}\left(N, T^{*} M\right)
$$

natural in $M$ and $N$. By the definition in Equation (30), the manifold $T^{*} N$ consists of the equivalence classes of tests in $\mathbb{R}^{N}$ indistinguishable by smooth paths in $N^{\mathbb{R}}$. On the other hand, two smooth morphisms $f, g \in \operatorname{Man}_{\infty}\left(M, M^{\prime}\right)$ that are indistinguishable by smooth paths in $M^{\prime}$ must be equal. So a morphism $f \in \operatorname{Man}_{\infty}\left(M, T^{*} N\right)$, unfolded to a function $\widehat{f}: M \times N \rightarrow \mathbb{R}$, is a representative of the equivalence classes modulo the same testing along each of the arguments. Although this function is not a manifold morphism (and the category $\operatorname{Man}_{\infty}$ is not cartesian closed), it is also a representative of the corresponding manifold morphism $f^{\prime} \in \operatorname{Man}_{\infty}\left(N, T^{*} M\right)$. 


\subsection{Smooth semantic connection}

The structures described so far form a semantic connection in the sense of Section 2 as follows:

- the category of smooth manifolds and smooth morphisms provides both the universe of 'spaces' to define systems, and the universe for 'types' to carry tests, i.e.

$$
\mathcal{S}=\mathcal{T}=\operatorname{Man}_{\infty}
$$

- the 'model/predicate' adjunction $M^{o p} \dashv P: \mathcal{S}^{o p} \rightarrow \mathcal{T}$ is given by the cotangent bundle construction

$$
\left(T^{*}\right)^{o p} \dashv T^{*}: \operatorname{Man}_{\infty}^{o p} \rightarrow \operatorname{Man}_{\infty},
$$

- the 'tests functor' $\Theta$ is the cotangent bundle functor $T_{\#}: \operatorname{Man}_{\infty} \rightarrow \operatorname{Man}_{\infty}$,

- the 'process functor' $\Sigma$ is the tangent bundle functor $T_{*}: \operatorname{Man}_{\infty} \rightarrow \operatorname{Man}_{\infty}$,

- the distributivity law $\lambda: \Theta P \rightarrow P \Sigma$ is derived as follows:

$$
\frac{\mathbb{R}^{T^{*}} \times T_{*} \stackrel{\text { id } \times \models}{\rightarrow} \mathbb{R}^{T^{*}} \times \mathbb{R}^{T^{*}} \stackrel{d}{\rightarrow} \mathbb{R}}{\lambda^{\prime}: T_{*} \rightarrow \mathbb{R}^{T^{T^{*}}} \rightarrow T^{*} T_{\#} T^{*}},
$$

where $d(x, y)=\|x-y\|$ is the distance on the manifold, and $=$ is the testing correlation between the tangents and the cotangents, induced as follows:

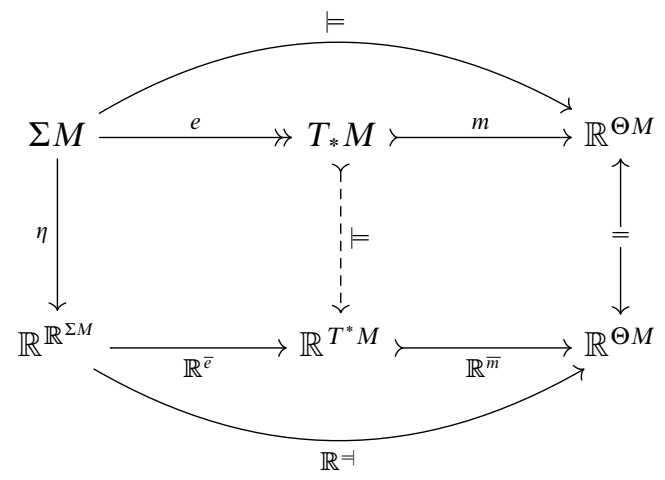

\section{Examples and applications}

5.1. Newtonian physics using coalgebras

We work in $\operatorname{Man}_{\infty}$ with smooth maps. A dynamical system modelled on $M$ is a coalgebra $X: M \rightarrow T * M$.

Definition 5.1. The model category Dyn of dynamical systems has as objects dynamical systems, that is smooth sections $X: M \rightarrow T_{*} M$ of the tangent bundle, and as morphisms 
$\left\langle f: M \rightarrow N, T_{*} f\right\rangle$ such that

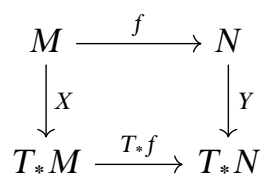

commutes. The dynamical systems $X$ and $Y$ are called $f$-related. The identity and composition are induced from $\operatorname{Man}_{\infty}$.

Dyn is the category of $T_{*}$-coalgebras $\operatorname{Man}_{T_{*}}$. The path category $\Sigma_{*}$ Man is a full subcategory of Dyn where the objects are in the form $R: I \rightarrow T_{*} I$ for open intervals $I \subset \mathbb{R}$. We have $R(t)=(t, 1)$ that is $\dot{x}=\mathrm{d} x / \mathrm{d} t=1$ represents a clock ${ }^{\dagger}$ at unit rate, setting a unit time interval. $T_{*} I \cong I \times \mathbb{R}$ is trivializable. We will assume that $I$ contains the point 0 .

Definition 5.2. A trajectory of a dynamical system is a morphism $\gamma: R \rightarrow X$ from the 'clock' morphism $R: I \rightarrow T I$ to the dynamical system $X: M \rightarrow T_{*} M$ such that

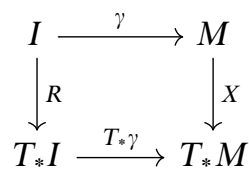

commutes.

Remark. $R$ describes the eigentime of a test particle (its internal clock) moving on the path. A suitable (monotone) map $R \mapsto R^{\prime}$ would describe the movement of the test particle with a different clock rate, hence points towards special relativity. We stick to classical mechanics here.

From $X \circ \gamma=T_{*} \gamma \circ R$, one obtains $(\gamma(t), \mathbf{D} \gamma(t) \cdot 1)=(\gamma(t), X(\gamma(t)) \cdot 1)$ and hence a first order differential equation $\dot{\gamma}(t)=X(\gamma(t))$ for $\gamma(t)$ with initial condition $q=\gamma(0)$. Such a curve is an integral curve. It can be integrated for some parameter ranges and initial conditions. The integral curve is unique on a maximal parameter range $I=(-a, a)$ (Kolar et al. 1993).

From coalgebras $x: X \rightarrow T_{*} X$, we get first order ordinary differential equations of the prototypical form $\dot{x}(t)=f(x(t))$. A formal solution is given by direct integration $x(t)=\int f(x(t))=\Phi(x(t))$. The integral operator is (circumstances permitting) a Fredholm operator and allows an iterative solution (resolvents and Neumann series). More prominently, a solution to the ordinary differential equation is a fix point for the integral operator $\Phi$, hence integral curves for a vector field (flow) are fix points.

5.1.1. Newtonian gravity. Our running example will be Newton's law of gravity. We use the following notations: $m$ is the mass (constant non-relativistic) of a body, $r$ its position with base point 0 in a Euclidean space $E=\mathbb{R}^{3} \backslash\{0\}, \hat{r}_{12}=r_{12} /\left|r_{12}\right|$ is the unit vector in

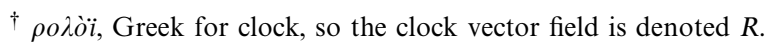


direction of $r_{12}, v=\dot{r}=\mathrm{d} r / \mathrm{d} t$ is the velocity of a body, $p=\mathrm{d}(m v) / \mathrm{d} t=m \dot{v}$ the (linear) momentum and $a=\dot{v}$ the acceleration. We put a source (mass) $m_{1}$ at the origin. The gravitational potential $\phi\left(r_{12}\right)=G \frac{m_{1}}{\left|r_{12}\right|}$ (potential energy per unit mass, zero at $\left|r_{12}\right|=\infty$ ) gives rise to the gravitational force ${ }^{\dagger} F\left(r_{12}\right)=\left\langle-\nabla m_{2} \phi\left(r_{12}\right), \hat{r}_{12}\right\rangle \hat{r}_{12}=-m_{2} \mathbf{D} \phi\left(r_{12}\right)$ on a test body $m_{2}$ at position $r_{12}$. Here, we use the relation between gradient and (directional) derivative $(\nabla f(x)) \cdot v=\left(\mathbf{D}_{v}\right) f(x)=\mathbf{D} f(x) \cdot v . F \in \Gamma T_{*} X$ is a section of the tangent bundle and assigns to each $r_{12}$ a linear curve ( $i_{U X}$ is the inverse of a trivial chart)

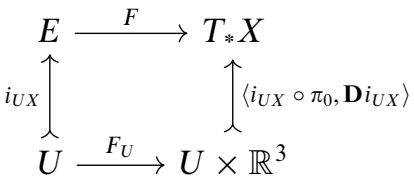

$$
\begin{aligned}
& F_{U}=\left\langle r_{12},-G \frac{m_{1} m_{2}}{\left|r_{12}\right|^{3}} r_{12}\right\rangle=\left\langle r_{12},-m_{2} \mathbf{D} \phi\left(r_{12}\right)\right\rangle
\end{aligned}
$$

5.1.2. Newton's second law. With the notation from above, Newtonian physics is based on three postulates:

NP1: For zero external force, any body moves on a straight line with constant velocity.

That is, $F=0 \Rightarrow \dot{v}=0$.

NP2: The change of momentum is equivalent to the external force acting on the body.

The law of motion is $\dot{p}=F$ with $p=m v$ and constant mass $(\dot{p}=m \dot{v})$.

NP3: For any two bodies $m_{i}, 1 \leqslant i \leqslant 2$, their interaction is such that the force $F_{12}(1$ acts on 2 ) and $F_{21}$ (2 acts on 1$)$ are summing to zero $F_{12}+F_{21}=0$. Action equals reaction.

Newton's second law implies

$$
\begin{aligned}
& \frac{\mathrm{d} r}{\mathrm{~d} t}=v \quad \frac{\mathrm{d} v}{\mathrm{~d} t}=\frac{1}{m} F(r) \quad \text { Lagrangian or configuration space view } \\
& \frac{\mathrm{d} r}{\mathrm{~d} t}=\frac{p}{m} \quad \frac{\mathrm{d} p}{\mathrm{~d} t}=F(r) \quad \text { Hamiltonian or phase space view. }
\end{aligned}
$$

The difference is that we look at different bundle structures. One can switch between these views taking into account the 'evaluation' (actually using the Hamiltonian and the symplectic form)

$$
T_{\#} M \times T_{*} M \rightarrow \mathbb{R}::(r, p) \times(r, v) \mapsto \frac{1}{2} p(v)=\frac{1}{2}\langle m v, v\rangle
$$

by the Riesz representation theorem. It involves the mass of the test body $m=m_{2}$ and describes the kinetic energy $E_{k i n}$ of the test body.

For Equation (43), consider the tangent bundle $F=\mathbb{R}^{3} \backslash\{0\} \times \mathbb{R}^{3}=T_{*} E$. Its points are states of the system $(r, v) \in T_{*}^{r} X$, and $T_{*} X$ is called configuration space. The configuration space is a manifold, and the law of motion induces a curve in it, that is we get (using the

\footnotetext{
$\dagger$ Technically, by Newton's third law (see below), the source $m_{1}$ is moved by the reaction force of the test mass $m_{2}$, and one uses the centre of mass and the reduced two body problem. If $m_{1} \gg m_{2}$ one can ignore this.
} 
names for maps in a local chart)

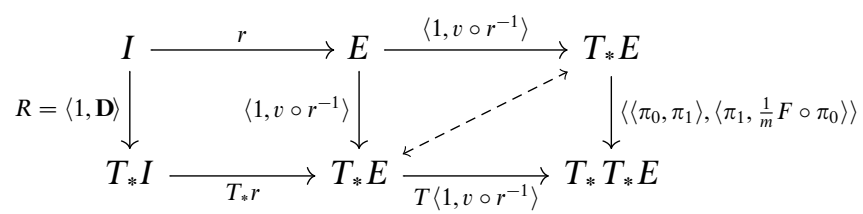

The commutativity of the left square $T_{*} r \circ R=\left\langle 1, v \circ r^{-1}\right\rangle \circ r$ gives $\left(r(t), \frac{\mathrm{d} r}{\mathrm{~d} t}(t)\right)=\left(T_{*} r \circ R\right)(t)=$ $\left(\left\langle 1, v \circ r^{-1}\right\rangle \circ r\right)(t)=(r(t), v(t))$, that is the LHS of Equation (43). The commutativity of the right square boils down to the commutativity of the lower triangle as the dashed arrow is identity. Hence, one gets $\left((r, v),\left(v, \frac{\mathrm{d} v}{\mathrm{~d} t}\right)\right)=\left(T_{*}\left\langle 1, v \circ r^{-1}\right\rangle\right)(r, v)=\left\langle\left\langle\pi_{0}, \pi_{1}\right\rangle,\left\langle\pi_{1}, \frac{1}{m} F \circ\right.\right.$ $\left.\left.\pi_{0}\right\rangle\right\rangle(r, v)=\left((r, v),\left(v, \frac{1}{m} F(r)\right)\right)$ and hence the RHS of Equation (43). A solution to this differential equation for the flow of the gravitational field is an integral curve in the double tangent bundle $F(r, v)=\left((r, v),\left(v, \frac{1}{m} F(r)\right)\right)$. The canonical flip acts trivially on this vector field.

For Equation (44), consider the cotangent bundle $T_{\#} E=\mathbb{R}^{3} \backslash\{0\} \times \mathbb{R}^{3}$. Its points are states of the system $(r, p) \in T_{\#}^{r} E$, and $T_{\#}^{r} E$ is called phase space. The phase space is a manifold, and the law of motion induces a curve in it, that is we get

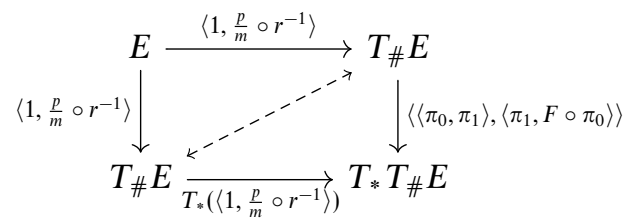

The corresponding vector field on phase space is given in (Abraham et al. 1988, p.240) and reads $F(r, p)=((r, p),(p / m, F(r))) \in T_{*} T_{\#} E$. Note that unlike for the double tangent bundle $T_{*}^{2} X$, we cannot expect the canonical flip to act here trivially as a duality $T_{*} X$ versus $T_{\#} X$ is involved.

\subsection{Other applications}

So far, this work focused on the correlations of tangents and cotangents over the same manifold. But the examples that arise in most applications involve two different manifolds: one to present the vector field, the other one to present the form on which it acts. Interesting questions that open up in this direction include:

5.2.1. Testing and monoidal (multilinear) structures. The example of Newtonian gravity in Section 5 describes one test particle in an external gravitational field of a source (mass). A natural question is to ask what happens when one looks at many particle systems. In such a situation, one needs to study paths $\gamma_{i}(t)$ parameterized over an index set $I$. That is, one looks at products of coalgebra maps each describing a path for a single particle in the presence of the others. Alternatively, one can study the path of a single system in a higher dimensional space. For example, two particles moving in $\mathbb{R}^{3}$ can be described as single system moving in $\mathbb{R}^{6}$. Categorically, this asks how the (co)tangent functors interact with sums and products $T_{*}(E+F)$ versus $T_{*} E \otimes T_{*} F$. Moreover, looking at the monad 
evaluation $T_{*} T_{*} M \stackrel{\mu}{\rightarrow} T_{*} M$ is given by the sum $\mu=+\circ u_{M}=+\circ\left\langle p_{T}, T_{*} p\right\rangle$ of the two projections from the double tangent bundle to the pullback bundle. The canonical flip, describing the commutativity of partial derivatives, and hence providing a local integrability condition, ensures that this can be done. What really is needed is a monoidal structure on the (co)tangent bundles which interacts properly with the lifting result of the Representation Theorem 2.1. This would allow to study the categorical backbone of $k$-forms for $k \geqslant 1$. This is important, as for example electromagnetism, and in general gauge theories, are based on (curvature) 2-forms, e.g. the Maxwell tensor $F$ is a closed 2-form.

5.2.2. Symplectic structure, general relativity. The testing framework yields in the differential geometry setting very naturally the tangent $T_{*}, T_{\#}$ and cotangent $T^{*}$ functors. On (finite) linear fiber spaces there is a closed structure available. However, in physical applications one uses also non-canonical identifications of these spaces.

A symplectic form is an isomorphism $\omega: T_{*} M \leftrightarrow T^{*} M: \Omega$. Using the closed structure, one has $\omega \in T^{*} M \wedge T^{*} M$. This 2-form plays a fundamental role in dynamical systems, e.g. transformations leaving this form invariant are symplectomorphisms inducing canonical transformations and do not change the nature of a physical system. The symplectic form provides also a description of time evolution of systems via the Hamiltonian $H$. Let $H: M \rightarrow \mathbb{R}$ be differentiable map, and consider the 1 -form $\mathrm{d} H$, which acts via the closed structure on $Y \in T_{*} M$. Then, one can define a Hamiltonian vector field $X_{H} \in T_{*} M$ via $\mathrm{d} H(Y)=\omega\left(X_{H}, Y\right)$. The Hamiltonian vector field describes the evolution of the system and implies the Hamiltonian form of the equations of motion. The interaction of the symplectic form, that is the closed structure, and the testing framework providing fixed point operators should allow to study general aspects of dynamical systems in a new way.

Given a (locally Minkowski) metric $g$ on the manifold allows to put a metric on the linear models $g: T_{*} M \times T_{*} M \rightarrow \mathbb{R}$. Via the Riesz representation theorem, this identifies also $T_{*} M$ and $T^{*} M$. We have seen that dynamical systems arise from the lifting of the paths functor $\Sigma$ to $T_{*}$-coalgebras. Hence, to make the metric a dynamical object, one needs a similar lifting for the situation in the diagram of the last paragraph with the metric identification of $T^{*}$ with $T_{*}$ and vice versa. Such a dynamic involves curvature as we are now dealing with higher forms, and it should be possible to employ the testing framework in a general relativistic setup. Note also that the distributivity law (39) depends on the distance function $d: \mathbb{R}^{T^{*}} \times \mathbb{R}^{T^{*}} \rightarrow \mathbb{R}$ allowing to include a metric.

5.2.3. Quantum mechanics. The testing framework does not choose bases, and also does not need in principle finite dimensional (co)tangent spaces. It is hence general enough to model infinite dimensional Hilbert spaces. Testing on such spaces can no longer be done point wise (Heisenberg uncertainty) and needs test functions. Using compactly supported or Schwartz test functions destroys the closed structure on the vector bundles as duals of such function spaces are strictly larger. Moreover, certain operators like the momentum operator are no longer bounded (continuous). Restricting the tests in the test category still allows to test systems, at the cost to be able to detect only such behaviour which 
is distinguishable by the chosen tests. One may expect that a coalgebraic study of this situation can reveal new insights not easy to gain by rather concrete coordinate based methods.

5.2.4. Scalings in chaotic systems and quantum field theory. Another way to employ coalgebraic methods in dynamical systems comes from studying fixed points and iterative solutions of processes. Following iteratively a tangent vector field, one obtains an approximation of integral curves (numerical integration). Suppose $f: E \rightarrow E$ is such a map. Let $f=f^{1}$ have a fixed point $f(a)=a$, and define $f^{n}=f \circ f^{n-1}$ the $n$-fold iteration of $f$. One defines formally the iteration velocity $v(x)=\partial f^{n}(x) /\left.\partial n\right|_{n=0}$. Let $g: E \rightarrow E$ be another function. In many applications, one is interested in the composition $g(f(x))$. Of course, $g(f(a))=g(a)$ for the fixed point. In non-linear dynamical systems and in quantum field theory at such fixed points, the system enjoys a scale invariance. However, apart from it the system changes behaviour for different scales. Using the iteration velocity as a (tangent) vector field, one obtains a solution for the composition problem

$$
g(f(x))=\exp \left(v(x) \frac{\partial}{\partial x}\right) g(x) .
$$

The flow of this vector field is a renormalization flow of the system. In quantum field, theory $v(x)$ is the beta-function and determines the 'running' (varying) coupling strength at a different energy scales. It seems plausible that techniques well established in computer science, e.g. final coalgebras etc, when employed via testing, could help studying these situations on a categorical level.

\section{Summary and future work}

Since its inception, category theory has played a crucial role in algebraic topology and algebraic geometry. There have many categorical approaches to vector calculus and to differential geometry, synthetic (Kock 2006) and classical (Frölicher and Kriegl 1988). There is a lot of current work, some of which we mentioned in the introduction. To the wealth of ideas and techniques resulting from all that work, we add a relatively minor conceptual wrinkle: semantical connections of vector fields and differential forms, and testing one over the other. On one hand, it is just a matter of presentation: we just proposed a coalgebraic reconstruction of standard concepts. This points towards a new realm of research in coalgebra, but it is not obvious that this research leads towards some genuinely new results in vector calculus or differential geometry. On the other hand, a high-level overview, abstracting away the irrelevant implementation details of a complicated mathematical theory can sometimes be even more useful than a particular mathematical result. This is not often the case, but it gave us a reason to pursue the ideas of smooth coalgebra, and of testing in vector analysis.

We started from the view of vector fields as coalgebras $M \rightarrow T_{*} M$ splitting the tangent bundle projection $T_{*} M \stackrel{\varepsilon}{\rightarrow} M$. This is just the coalgebraic form of the familiar cross-section requirement, which assures that the corresponding vector fields are locally determined. Coalgebras not satisfying this requirement permit non-local interactions. Differential 
forms similarly arise as the crossections $M \rightarrow T_{\#} M$ of the cotangent bundle projection $T_{\#} M \stackrel{\varepsilon}{\rightarrow} M$. The Main Theorem opened up the alley towards testing vector fields and differential forms. But the actual explorations of that alley are left for the future work.

So what did we actually do here? We defined the sheaves $\Sigma M$ and $\Theta M$, consisting respectively of the paths and of the tests over a manifold $M$, and established the canonical testing correlation between them $\Sigma M \times \Theta M \stackrel{\mathbb{T}}{\rightarrow} \mathbb{R}$, mapping a path $\varsigma: \mathbb{R} \rightarrow M$ and a test $t: M \rightarrow \mathbb{R}$ to $\mathbf{D}(t \circ \varsigma)(0)$. Applying the testing factorization (5) to $\mathbb{T}$, we then derived the tangent bundle $T_{*} M$, as the sheaf of paths indistinguishable under the tests, and the cotangent bundle functor $T^{*} M$, as the sheaf of tests indistinguishable under the paths. As a byproduct of the construction, the testing correlation $T_{*} M \times T^{*} M \stackrel{\mathbb{T}}{\rightarrow} \mathbb{R}$ emerged in Equation (40), embedding $T_{*} M \hookrightarrow \mathbb{R}^{T^{*} M}$ and $T^{*} M \hookrightarrow \mathbb{R}^{T_{*} M}$. Uncovering a duality of $T_{*} M$ and $T^{*} M$ is in itself, of course, hardly surprising. Uncovering it through a testing correlation, akin to the 'Gedankenexperiments' whereby Turing machines give birth to complexity classes, is perhaps more interesting. Where does it lead?

In Section 4.5, we outlined a possible second application of the testing factorization (5), towards a coalgebraic framework for describing the families of smooth behaviours indistinguishable under a family of smooth tests. This leads towards a coalgebraic extension of Mackey's dual systems (Mackey 1945), studied in detail in Grothendieck (1973, Ch. 2-3), and axiomatized in Chu categories (Barr 1979, 2006). While the testing correlations considered in this paper were limited to the tangents and the cotangents on the same manifold $M$, the correlations considered in the practice are often between the tangents on one manifold, and the cotangents on another one. An interesting framework to further research in this direction seems to be the category STC, where

- the objects are the smooth testing correlations

$$
\alpha=\left(\alpha_{*} \times \alpha_{\#} \stackrel{\mathbb{T}}{\rightarrow} \mathbb{R}\right),
$$

where

- $T_{*} M \stackrel{\alpha_{*}}{\rightarrow} M$ is an algebra,

- $N \stackrel{\alpha_{\#}}{\longrightarrow} T_{\#} N$ is a coalgebra, and

- the testing correlations $\mathbb{T}$ make the following square commute

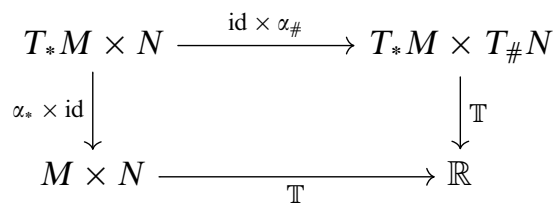

- a morphism $f: \alpha \rightarrow \beta$ is a pair $f=\left\langle f_{*}, f_{\#}\right\rangle$, where

- $\alpha_{*} \stackrel{f_{*}}{\longrightarrow} \beta_{*}$ is an algebra homomorphism,

- $\beta_{\#} \stackrel{f_{\#}}{\longrightarrow} \alpha_{\#}$ is a coalgebra homomorphism, and 
- the following square commutes

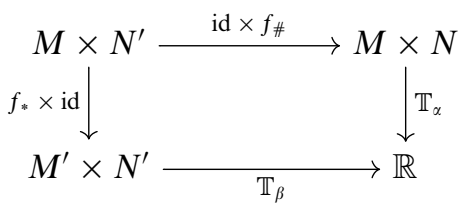

where $M^{\prime}$ is the underlying object of $\beta_{*}$ and $N^{\prime}$ is the underlying object of $\beta_{\#}$.

The morphisms between certain integral operators as testing correlations express the Stokes-type of theorems (including the Fundamental Theorem of Calculus). The behaviours observable through such testing correlations can now be extracted by factoring out the separable and extensional objects of the category STC (Barr 1996, Section 2). The various algebraic invariants arise from manifolds through testing vector fields, just like the classes of languages arise through testing state machines. The question is whether this conceptual link is strong enough to support any technical hooks.

We are grateful to the editors and to the anonymous reviewers for their patience and help. Some of the high-level ideas pursued in this work were suggested to D. Pavlovic in a phone conversation with Bill Lawvere some 20 years ago, following the first author's talk about Chu spaces, later presented in Pavlovic (1997). It took him almost 10 years to overcome the excitement about the call, and to understand what was suggested. The framework presented here subsequently evolved through a variety of concrete, domainspecific applications in various research projects (on hybrid systems, control theory, embedded systems); and it took another 10 years for an occasion to arise for this general overview. At best, the present paper brings us in the position to begin to study the original idea, sketched in the final section. While an elaboration in cartesian closed categories, along the lines of Lawvere (1980), would surely have its advantages, we felt that it is important to tell the story in a language as standard as possible, as it may be of interest for several different communities (coalgebraists, physicists, functional analysts ... ). The balance of a presentation is, of course, always a matter of skill and taste, and we can only hope that our clumsiness will not completely obscure the clarity of the ideas that we attempted to present.

\section{Appendix A. Norms and vector spaces}

Manifolds and their relations to differential geometry and sheaves are well studied. In preparing the paper, we used the books by Mac Lane and Moerdijk (1992), and by Abraham et al. (1988). The statements given without proof are proved in these books. For further information about invariant operations in differential geometry see Kolar et al. (1993). Smoothness allows to approximate functions and such approximations are characterized using norms (or if available inner products).

Definition A.1. A norm of a real vector space $E$ is a mapping from $E$ into the reals $\|\cdot\|: E \rightarrow \mathbb{R}:: e \mapsto\|e\|$ such that 
$\mathrm{N} 1\|e\| \geqslant 0$ for all $e \in E$, and $\|e\|=0$ if and only if $e=0$ (positive definiteness).

$\mathrm{N} 2\|\lambda e\|=|\lambda|\|e\|$, for all $e \in E$ and $\lambda \in \mathbb{R}$ (multiplicativity).

N3 $\left\|e_{1}+e_{2}\right\| \leqslant\left\|e_{1}\right\|+\left\|e_{2}\right\|$ for all $e_{1}, e_{2} \in E$ (triangle inequality).

$(E,\|\cdot\|)$ is a normed space. A norm complete space is called a Banach space.

A more special situation arises in presence of an inner product.

Definition A.2. A real inner product space is a real vector space $E$ together with a mapping $\langle\cdot, \cdot\rangle: E \times E \rightarrow \mathbb{R}::\left(e_{1}, e_{2}\right) \mapsto\left\langle e_{1}, e_{2}\right\rangle$ such that

$11\left\langle e, e_{1}+e_{2}\right\rangle=\left\langle e, e_{1}\right\rangle+\left\langle e, e_{2}\right\rangle$, (linearity, part 1$)$.

$12\left\langle e_{1}, \lambda e_{2}\right\rangle=\lambda\left\langle e_{1}, e_{2}\right\rangle$, (linearity, part 2).

$13\left\langle e_{1}, e_{2}\right\rangle=\left\langle e_{2}, e_{1}\right\rangle$, (symmetry).

$14\langle e, e\rangle \geqslant 0$ and $\langle e, e\rangle=0$ if and only if $e=0$ (positive definiteness).

Positive definiteness provides a non-natural isomorphism $E \rightarrow \mathbb{R}^{E} \cong E^{*}$ with the dual vector space $E^{*}$. If such an isomorphism exists the space is called reflexive. A complete (w.r.t. a metric) reflexive inner product space is called Hilbert space.

The complex case does not pose any difficulty here, and one defines Hermitian inner products using the complex involution analogously. An inner product space is a normed space by setting $\|e\|^{2}=\langle e, e\rangle$.

Definition A.3. A metric or distance map on a set $E$ is a map $d_{E}: E \times E \rightarrow \mathbb{R}$ such that $\mathrm{d} 1 d(x, y) \geqslant 0$ non-negativity or separation axiom.

$\mathrm{d} 2 d(x, y)=0 \Leftrightarrow x=y$, coincidence axiom (if not fulfilled $d_{E}$ is called pseudo metric).

d3 $d(x, y)=d(y, x)$ symmetry (if not fulfilled $d_{E}$ is called quasi metric).

$\mathrm{d} 4 d(x, z) \leqslant d(x, y)+d(y, z)$ triangle inequality.

d1 and d2 state positive definiteness. An intrinsic metric $d_{M}$ on a manifold $M$ can be approximated by the length of paths connecting two points $x=\gamma(0), y=\gamma(1)$ and $d_{M}(x, y)=\inf _{\gamma}$ length $(\gamma)$. Suppose $E$ has an addition $+: E \times E \rightarrow E$, e.g. a vector space structure, then $d$ is translation invariant if for all $x, y, a \in E$ holds $d(x, y)=d(x+a, y+a)$. Any norm implies a metric by $d(x, y):=\|y-x\|$. A metric is homogeneous if for $r \in \mathbb{R}$ and all $x, y \in E$ holds $d(r x, r, y)=|r| d(x, y)$. A translation invariant homogeneous metric defines a norm $\|x\|:=d(x, 0)$.

Theorem A.4 (Riesz representation theorem). Let $E$ be an real inner product space. The map $e \mapsto\langle\cdot, e\rangle$ is a linear norm preserving isomorphism of $E$ with $E^{*}$.

In other words, every linear form in $E^{*}$ can be realized as an inner product with some fixed vector $e \in E$. We denote by $L(E, F)$ the linear morphisms from $E$ to $F$.

\section{Appendix B. Differential}

If $f: U \subset \mathbb{R}^{n} \rightarrow \mathbb{R}^{m}$ is differentiable, then choosing coordinates (bases), gives $f$ explicit as an $m$-tuple of functions in $n$ coordinates $\left(y_{1}=f^{1}\left(x_{1}, \ldots, x_{n}\right), \ldots, y_{m}=f^{m}\left(x_{1}, \ldots, x_{n}\right)\right)$. 
The differential $\mathbf{D} f(x)$ at a point $x_{0} \in U$ is then given by the Jacobian matrix $J(f) \simeq \mathbf{D} f$

$$
\text { D } f\left(x_{0}\right):=\left[\begin{array}{ccc}
\frac{\partial f^{1}(x)}{\partial x_{1}} & \cdots & \frac{\partial f^{1}(x)}{\partial x_{n}} \\
\vdots & & \vdots \\
\frac{\partial f^{m}(x)}{\partial x_{1}} & \cdots & \frac{\partial f^{m}(x)}{\partial x_{n}}
\end{array}\right]_{x=x_{0}} \text {. }
$$

For $m=1$, one has $y=f\left(x_{1}, \ldots, x_{n}\right)$ and the differential becomes

$$
\mathbf{D} f=\left[\begin{array}{lll}
\frac{\partial f}{\partial x_{1}} & \cdots & \frac{\partial f}{\partial x_{n}}
\end{array}\right], \quad \text { applied to } e=\left(e^{1}, \ldots, e^{n}\right)^{t} \quad \mathbf{D} f(x) \cdot e=\sum_{i=1}^{n} \frac{\partial f}{\partial x_{i}} e^{i} .
$$

As an example for an iterated differential $\mathbf{D}^{2} f \in L^{2}\left(\mathbb{R}^{n}, \mathbb{R}\right) \cong L\left(\mathbb{R}^{n} \otimes \mathbb{R}^{n}, \mathbb{R}\right)$, with $f: U \subset$ $\mathbb{R}^{n} \rightarrow \mathbb{R}$ one obtains

$$
\mathbf{D}^{2} f\left(x_{0}\right)=\left[\frac{\partial^{2} f(x)}{\partial x_{i} \partial x_{j}}\right]_{x=x_{0}} .
$$

From this, it is easy to conclude that the differential is a linear operator on the space of sufficiently often differentiable functions.

The tangent construction $T_{*}$ is algebraically and geometrically simpler that $\mathbf{D}$. Think of $(u, e)$ as a vector $e$ in $E$ with base point $u$, then $T_{*} f(u, e)=(f(u), \mathbf{D} f(u) \cdot e)$ is the image vector in $F$ with base point $f(u)$. The map $T_{*}$ is functorial while $\mathbf{D}$ suffers from a morphism present to change the base point successively. To see this, let $f: U \subset E \rightarrow F$ and $g: V \subset F \rightarrow G$ be composable $C^{1}$ maps, then we get the

Theorem B.1. (Composite mapping theorem) With $f, g$ as above, $g \circ f: U \subset E \rightarrow G$ is also $C^{1}$. Moreover,

$$
\begin{aligned}
T_{*}(g \circ f) & =T_{*}(g) \circ T_{*}(f) \\
\mathbf{D}(g \circ f)(u) & =\mathbf{D} g(f(u)) \cdot \mathbf{D} f(u) \quad \text { (chain rule). }
\end{aligned}
$$

The equation for $\mathbf{D}$ is the chain rule and reads in coordinates as follows:

$$
\frac{\partial(g \circ f)^{j}(x)}{\partial x^{i}}=\sum_{k=1}^{n} \frac{\partial g^{j}(f(x))}{\partial y^{k}} \frac{\partial f^{k}(x)}{\partial x^{i}} \quad i=1, \ldots, m, \quad y^{k}=f^{k}(x) .
$$

The chain rule implies the Leibniz rule if applied to $B \in L\left(F_{1} \otimes F_{2}, G\right)$ and map $f_{1} \times f_{2}$ : $U \times U \rightarrow F_{1} \times F_{2}$.

Theorem B.2. (Leibniz rule) Let $f_{i}: U \subset E \rightarrow F_{i}$ for $i=1,2$ be of class $C^{1}$, and $B \in L\left(F_{1} \otimes F_{2}, G\right)$. Then, the mapping $B\left(f_{1}, f_{2}\right)=B \circ\left(f_{1} \times f_{2}\right): U \subset E \rightarrow G$ is of class $C^{1}$ and

$$
\mathbf{D}\left(B\left(f_{1}, f_{2}\right)\right)(u) \cdot e=B\left(\mathbf{D} f_{1}(u) \cdot e, f_{2}(u)\right)+B\left(f_{1}(u), \mathbf{D} f_{2}(u) \cdot e\right) .
$$

For one dimensional real vector spaces, $B$ is just multiplication in $\mathbb{R}$, hence $\mathbf{D}\left(f_{1} f_{2}\right)(u)=$ $\mathbf{D}\left(f_{1}(u)\right) f_{2}(u)+f_{1}(u) \mathbf{D} f_{2}(u)$, where $e=1$ is the unit vector tangent to the real line.

The tangent map can be applied to itself, hence one can define the second tangent map $T_{*}^{2} f=T_{*}\left(T_{*} f\right)$. Let $f: U \subset E \rightarrow F$, then the second tangent map is given locally in 
terms of differentials as

$$
\begin{aligned}
& T_{*}^{2} f:(U \times E) \times(E \times E) \rightarrow(F \times F) \times(F \times F) \\
& \quad\left(u, e_{1}, e_{2}, e_{3}\right) \mapsto\left(f(u), \mathbf{D} f(u) \cdot e_{1}, \mathbf{D} f(u) \cdot e_{2}, \mathbf{D}^{2} f(u) \cdot\left(e_{1}, e_{2}\right)+\mathbf{D} f(u) \cdot e_{3}\right) .
\end{aligned}
$$

To see this, recall that

$$
T_{*} f(u, e)=(f(u), \mathbf{D} f(u) \cdot e) .
$$

Applying $T_{*}$ to $T_{*} f$ yields

$$
\begin{aligned}
T_{*}\left(T_{*} f\right)\left(u, e_{1}, e_{2}, e_{3}\right) & =\left(T_{*} f\left(u, e_{1}\right), \mathbf{D}\left(T_{*} f\right)\left(u, e_{1}\right) \cdot\left(e_{2}, e_{3}\right)\right) \\
& =\left(f(u), \mathbf{D} f(u) \cdot e_{1}, \mathbf{D}\left[\left(f(u), \mathbf{D} f(u) \cdot e_{1}\right)\right] \cdot\left(e_{2}, e_{3}\right)\right) \\
& =\left(f(u), \mathbf{D} f(u) \cdot e_{1}, \mathbf{D} f(u) \cdot e_{2}, \mathbf{D}^{2} f(u) \cdot\left(e_{1}, e_{2}\right)+\mathbf{D} f(u) \cdot e_{3}\right),
\end{aligned}
$$

where in the last equality the identity $\mathbf{D}[1]=\pi_{1}$ has been used and the Leibniz rule was applied.

To finish the setup, we consider differentials in a particular direction. A differentiable path is a class $C^{1}$ map from an open interval $\gamma: I \subset \mathbb{R} \rightarrow E$ into $E$, with differential $\mathbf{D} \gamma(t) \in L(I, E) . L(\mathbb{R}, E)$ is identified with $E$ via $\mathbf{D} \gamma(t)$ acting on $e=1$. The differential of the curve is

$$
\frac{\mathrm{d} \gamma}{\mathrm{dt}}(t)=\mathbf{D} \gamma(t) \cdot 1
$$

Composing a $C^{1}$ curve $\gamma: I \rightarrow E$ with a $C^{1}$ map $f: E \rightarrow F$ and using the chain rule yields

$$
\frac{\mathrm{d}}{\mathrm{dt}}(f(\gamma(t)))=\mathbf{D}(f \circ \gamma)(t) \cdot 1=\mathbf{D} f(\gamma(t)) \cdot \frac{\mathrm{d} \gamma}{\mathrm{dt}}(t)
$$

Using the linear approximation $\gamma_{L}(t)=u+t \cdot e$ of $\gamma(t)$, one arrives at the

Theorem B.3. Let $f: U \subset E \rightarrow F$ be of $C^{1}$ differentiable at $u$, then the directional differential exists at $u$ and reads

$$
\left.\frac{\mathrm{d}}{\mathrm{dt}}(f(u+t \cdot e))\right|_{t=0}=\mathbf{D} f(u) \cdot e=\mathbf{D}_{e} f(u) .
$$

If $f: \mathbb{R}^{n} \rightarrow \mathbb{R}$ with coordinate (basis) functions $x^{i}$ and let $e=x^{1} e_{1}+\cdots+x^{n} e_{n}$ (usually normalized) expressed in the standard basis $\left\{e_{i}\right\}$, then the directional differential (in one or many directions $v_{i}$ ) reads

$$
\begin{aligned}
\mathbf{D} f(u) \cdot e=\mathbf{D}_{e} f(u) & =\frac{\partial f}{\partial x^{1}} x^{1}+\cdots+\frac{\partial f}{\partial x^{n}} x^{n} \\
\mathbf{D}_{v_{1}, \ldots, v_{k}}^{k} f(u) & =\left.\frac{\partial^{k}}{\partial t_{1} \cdots \partial t_{k}} f\left(u+t_{1} v_{1}+\cdots+t_{k} v_{k}\right)\right|_{t_{i}=0} .
\end{aligned}
$$

As we compare linearizations, we can replace the open interval $I$ by $\mathbb{R}$ if the reparameterization is done in such a way that base points and tangents are preserved. 


\section{References}

Abraham, R., Marsden, J. and Ratiu, T. (1988). Manifolds, Tensor Analysis, and Applications. Applied Mathematical Sciences, volume 75, Springer-Verlag, New York.

Aczel, P. (1988). Non-Well-Founded Sets. Number 14 in Lecture Notes. Center for the Study of Language and Information, Stanford University.

Artin, M., Grothendieck, A. and Verdier, J. (1972). Théorie des topos et cohomologie étalé des schémas, Lecture Notes in Mathematics, volume 269, 270, 305, Springer-Verlag.

Barr, M. (1979). *-Autonomous Categories, Lecture Notes in Mathematics, 752, Springer-Verlag.

Barr, M. (1996). *-autonomous categories, revisited. Journal of Pure and Applied Algebra 111 (1) $1-20$.

Barr, M. (2006). The chu construction: history of an idea. Theory and Application of Categories 17 (1) $10-16$.

Bernardo, M., De Nicola, R. and Loreti, M. (2012). Revisiting trace and testing equivalences for nondeterministic and probabilistic processes. In: Birkedal, L. (ed.) Proceedings of FOSSACS 2012. Lecture Notes in Computer Science 7213, Springer, Berlin, 195-209.

Blute, R., Cockett, J.R.B. and Seely, R.A.G. (2006). Differential categories. Mathematical Structures in Computer Science 16 (6) 1049-1083.

Blute, R., Cockett, J.R.B. and Seely, R.A.G. (2009). Cartesian differential categories. Theory and Applications of Categories 22 (23) 622-672.

Caenepeel, S., Militaru, G. and Zhu, S. (2002). Frobenius and Separable Functors for Generalized Module Categories and Nonlinear Equations, Springer-Verlag.

Cockett, J.R.B. and Cruttwell, G.S.H. (2014). Differential structure, tangent structure, and SDG. Applied Categorical Structures 22 (2) 331-417.

Cockett, J.R.B. and Seely, R.A.G. (2011). The Faà di Bruno construction. Theory and Application of Categories 25 (15) 393-425.

De Nicola, R. and Hennessy, M. (1984). Testing equivalences for processes. Theoretical Computer Science 34 (1-2) 83-133.

Dold, A. (1995). Lectures on Algebraic Topology, Classics in Mathematics, Springer, Berlin, Heidelberg.

Frölicher, A. and Kriegl, A. (1988). Linear Spaces and Differentiation Theory, Pure and Applied Mathematics, Wiley.

Grothendieck, A. (1973). Topological Vector Spaces, Notes on mathematics and its applications, Gordon and Breach.

Haghverdi, E., Tabuada, P. and Pappas, G.J. (2005). Bisimulation relations for dynamical, control, and hybrid systems. Theoretical Computer Science 342 (2-3) 229-261.

Harel, D., Tiuryn, J. and Kozen, D. (2000). Dynamic Logic, MIT Press, Cambridge, MA, USA.

Hasuo, I., Jacobs, B. and Sokolova, A. (2007). Generic trace semantics via coinduction. Logical Methods in Computer Science 3 (4).

Jackson, A. (2004). Comme Appelé du Néant - as if summoned from the void: The life of Alexandre Grothendieck. Notices of the AMS $51(4,10)$ 1038-1056, 1196-1212.

Jacobs, B. and Sokolova, A. (2010). Exemplaric expressivity of modal logics. Journal of Logic and Computation 20 (5) 1041-1068.

Jubin, B. t. M. (2012). The Tangent Functor Monad and Foliations. PhD thesis, University of Berkeley, Berkeley. arxiv:1401.0940.

Kapulkin, K., Kurz, A. and Velebil, J. (2012). Expressiveness of positive coalgebraic logic. In: Bolander, T., Braüner, T., Ghilardi, S. and Moss, L. S. (eds.) Advances in Modal Logic, College Publications 368-385. 
Klin, B. (2007a). Bialgebraic operational semantics and modal logic. In: LICS, IEEE Computer Society $336-345$.

Klin, B. (2007b). Coalgebraic modal logic beyond sets. Electronic Notes in Theoretical Computer Science 173 177-201.

Klin, B. (2009). Bialgebraic methods and modal logic in structural operational semantics. Information and Computation 207 (2) 237-257.

Kock, A. (2006). Synthetic Differential Geometry, volume 333 of London Mathematical Society Lecture Note Series. Cambridge University Press.

Kolar, I., Michor, P. and Slovak, J. (1993). Natural Operations in Differential Geometry, SpringerVerlag.

Kupke, C., Kurz, A. and Pattinson, D. (2004). Algebraic semantics for coalgebraic logics. Electronic Notes in Theoretical Computer Science 106 219-241.

Kupke, C., Kurz, A. and Pattinson, D. (2005). Ultrafilter extensions for coalgebras. In: et al, J. L. F. (eds.) CALCO. Lecture Notes in Computer Science 3629, Springer, Berlin, 263-277.

Kurz, A. and Rosický, J. (2012). Strongly complete logics for coalgebras. Logical Methods in Computer Science 8 (3) 1-32.

Lawvere, F.W. (1980). Toward the description in a smooth topos of the dynamically possible motions and deformations of a continuous body. Cahiers de Topologie et Gomtrie Diffrentielle Catgoriques 21 (4) 377-392.

Lawvere, W.F. (1975). Continuously variable sets: Algebraic geometry = geometric logic. In: Logic Colloquium '73 (Bristol, 1973). Studies in Logic and the Foundations of Mathematics 80, NorthHolland, Amsterdam 135-156.

Lawvere, W.F. (1986). Categories of spaces may not be generalized spaces as exemplified by directed graphs. Revista Colombiana de Matematicas 20 179-186.

Leray, J. (1945). Sur la forme des espaces topologiques et sur les pointes fixes des représentations. Journal de Mathématiques Pures et Appliquées 9 95-249.

Mac Lane, S. and Moerdijk, I. (1992). Sheaves in Geometry and Logic: A First Introduction to Topos Theory, Universitext. Springer-Verlag, New York.

Mackey, G. (1945). On infinite dimensional vector spaces. Transactions of the American Mathematical Society 51 (57) 155-207.

Mesablishvili, B. and Wisbauer, R. (2010). Galois functors and entwining structures. J. Algebra 324 464-506.

Mesablishvili, B. and Wisbauer, R. (2011). Bimonads and Hopf monads on categories. Journal of K-Theory 7 (2) 349-388.

Milner, R. (1989). Communication and Concurrency, International Series in Computer Science, Prentice Hall, London.

Modungo, M. and Stefani, G. (1978). Some results on second tangent and cotangent spaces. Quaderni dell'Instituto di Matematica dell' Universitá di Lecce Q. 16 1-23.

Moerdijk, I. (2002). Monads on tensor categories. Journal of Pure and Applied Algebra 168 (2-3) 189-208.

Moore, E. (1956). Gedanken experiments on sequential machines. In: Shannon, C. E. and McCarthy, J. (eds.) Automata Studies, Annals of Mathematics Studies, Princeton, Princeton University Press $129-153$.

Pavlovic, D. (1995). Convenient categories of processes and simulations I: Modulo strong bisimilarity. In: Pitt, D., Rydeheard, D.E. and Johnstone P. (eds.) Category Theory and Computer Science '95. Lecture Notes in Computer Science 953, Springer-Verlag, Berlin, 3-24.

Pavlovic, D. (1997). Chu i: Cofree equivalences, dualities and *-autonomous categories. Mathematical Structures in Computer Science 7 (2) 49-73. 
Pavlović, D. (2001). Towards semantics of self-adaptive software. In Robertson, P., Shrobe, H. and Laddaga, R. (eds.) Proceedings of the Workshop on Self-Adaptive Software. Lecture Notes in Computer Science 1936, Springer-Verlag, Berlin, 50-64.

Pavlovic, D. (2013). Monoidal computer I: Basic computability by string diagrams. Information and Computation 226 94-116.

Pavlovic, D. and Escardó, M. (1998). Calculus in coinductive form. In: Pratt, V. (eds.) Proceedings 13th Annual IEEE Symposium on Logic in Computer Science, IEEE Computer Society 408-417.

Pavlovic, D., Mislove, M. and Worrell, J. (2006). Testing semantics: Connecting processes and process logic. In: Johnson, M. and Vene, V. (eds.) AMAST 2006. Springer-Verlag Lecture Notes in Computer Science 4019 308-322 Berlin. (The version with the Appendix is available from dusko.org).

Pavlovic, D., Pepper, P. and Smith, D.R. (2008). Evolving specification engineering. In: Meseguer, J. and Rosu, G. (eds.) Proceedings of AMAST 2008. Springer Verlag Lecture Notes in Computer Science 5140 299-314.

Pavlovic, D., Pepper, P. and Smith, D.R. (2010). Formal derivation of concurrent garbage collectors. In: Desharnais, J. (ed.) Proceedings of MPC 2010. Springer Verlag Lecture Notes in Computer Science 6120 353-376. full version arxiv.org:1006.4342.

Pavlovic, D. and Smith, D.R. (2001). Composition and refinement of behavioral specifications. In: The 16th International Conference on Automated Software Engineering, IEEE.

Pavlovic, D. and Smith, D. R. (2002). Guarded transitions in evolving specifications. In: Kirchner, H. and Ringeissen, C. (eds.) Proceedings of AMAST 2002. Springer Verlag Lecture Notes in Computer Science 2422 411-425.

Pratt, V.R. (1976). Semantical consideration on Floyd-Hoare logic. In: Proceeding of the 17th Annual Symposium on Foundations of Computer Science, IEEE 109-121.

Rosický, J. (1984). Abstract tangent functors. Diagrammes 12 (3) JR1-JR11.

Rutten, J. (2000). Universal coalgebra: A theory of systems. Theoretical Computer Science 249 3-80.

Schwartz, L. (1950). Théorie des Distributions, Hermann, Paris.

Tennison, B. (1975). Sheaf Theory, Cambridge Monographs on Physics, Cambridge University Press.

Turi, D. and Plotkin, G.D. (1997). Towards a mathematical operational semantics. In: LICS, IEEE Computer Society, 280-291.

van Glabbeek, R.J. (1990). The linear time-branching time spectrum (extended abstract). In: Baeten, J.C.M. and Klop, J.W. (eds.) Proceedings of CONCUR '90. Springer Lecture Notes in Computer Science 458 278-297.

van Glabbeek, R.J. (1993). The linear time - branching time spectrum II. In: Best, E. (eds.) Proceedings of CONCUR '93. Springer Lecture Notes in Computer Science $\mathbf{7 1 5 .}$ 